\title{
Decomposition of Wavelength Dispersive $X$-Ray Spectra
}

\section{Guy Rémond}

Australian Key Centre for Microscopy and Microanalysis, The University of Sydney, NSW 2006, Australia

and

Laboratoire de Microanalyse des Surfaces,

Ecole Nationale de Mécanique et des Microtechniques,

Besançon, France

\section{Robert Myklebust}

National Institute of Standards and Technology,

Gaithersburg, MD 20899-0001

\section{Michel Fialin}

Ura 736-CNRS,

Université $\mathrm{P}$ et $\mathrm{M}$ Curie,

Paris, France

\section{Clive Nockolds}

Electron Microsco Unit, The University of Sydney,

NSW 2006, Australia

\section{Matthew Phillips}

Microstructural Analysis Unit, University Technology of Sydney, NSW 2007, Australia

and

\section{Claude Roques-Carmes}

Laboratoire de Microanalyse des Surfaces,

Ecole Nationale de Mécanique et des Microtechniques,

\section{Besançon, France}

\section{guy.remond@net-up.com} rmykleb@fred.net fialin@ccr.jussieu.fr clive@emu.usyd.edu.au matthew.phillips@uts.edu.au Ims-sec@ens2m.fr

\begin{abstract}
Line shapes of atomic lines and soft $\mathrm{x}$-ray emission bands measured with a wavelength dispersive spectrometer (WDS) with the Electron Probe Micro Analyzer (EPMA) are reviewed. Least square fitting to pseudo-Voigt profiles of the digitally measured spectra are used to account for the presence of non-diagram features (high and low energy satellites) and instrumental induced distortions. The effect of line width and relative intensities on the quality of fits is illustrated. Spectral distortions resulting from the presence of absorption edges within the analyzed wavelength region are illustrated for the case of FeL $\alpha, \beta$ emission bands for pure $\mathrm{Fe}$ and iron oxides. For quantitative analysis, an analytical approach is presented where the measured soft $\mathrm{x}$-ray emission bands are corrected for self absorption before extracting the intensities from the experimental data.
\end{abstract}

Key words: atomic lines; distortions induced by absorption edges; pseudo-Voigt profiles; satellites; soft $\mathrm{x}$-ray bands; WDS instrumental distortions.

Accepted: August 22, 2002

Available online: http://www.nist.gov/jres

\section{Contents}

1. Introduction ................... 510

2. Contributions of X-Ray Generation Mechanisms and Instrumental Factors to the Shape of a WDS X-Ray Line........ 510

2.1 The Diagram Lines ................ 510

2.2 The Non-Diagram Lines ............. 511

2.2.1 High Energy Satellites ......... 511
2.2.2 Low Energy Satellites ......... 513

2.3 Instrumental Distortions.............. 514

3. Deconvolution vs Spectral

Decomposition Using Least-Square Fitting

Techniques...................... 517

3.1 A Need for a Unique Approach for

EDS and WDS Spectra Processing..... 518 
3.2 The Fitting Function to Mono-Energetic Spectroscopic Features ........... 518

3.3 The Fitting Function to the Continuous Emission Distribution. .......... 518

4. Applications of Least-Square Fitting

Techniques to WDS Spectra . . . . . . . . . 520

4.1 Practical Considerations. . . . . . . . . . . . . 520

4.2 Gaussian to Lorentzian Proportion and Line Widths. . . . . . . . . . . . . 520

4.3 Relative Intensities . . . . . . . . . . . . . . 522

4.4 Peak Shape Modifications Related to the Presence of Absorption Edges . . . . . 524 4.4.1 Fe $\mathrm{L} \alpha, \beta$ Emission Bands for Pure Iron. . . . . . . . . . . . . . . 524

4.4.2 Fe L $\alpha, \beta$ Emission Bands From Iron Oxides. . . . . . . . . . . . 525

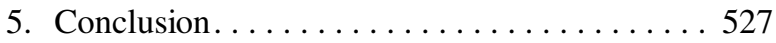

6. References......... . . . . . . . . . 527

\section{Introduction}

It is well recognized that the peak height of an x-ray emission line measured with a wavelength dispersive spectrometer (WDS) is a sufficient approximation for quantitative microanalysis on a routine basis using an Electron Probe Micro Analyzer (EPMA). This approach assumes that the observed $\mathrm{x}$-ray line is symmetrical around the peak maximum occurring at a Bragg angle characteristic of the analyzed emission. A symmetrical peak never exists even for the case of atomic lines resulting from radiative transitions involving only core levels because of the presence of high and low energy satellites and instrumental distortions induced during measurement. While the approximation of the peak height for the $\mathrm{x}$-ray intensity measurement remains valid in most analytical problems with the EPMA, this simplified approach is no longer sufficient in the presence of severe peak overlaps as is the case for L emission spectra of rare-earth elements [1] or when soft x-ray emissions are used in the analytical procedure [2-4].

We define soft $\mathrm{x}$-ray emission as $\mathrm{x}$-ray emission with energies lower than $1 \mathrm{keV}$ such as the $\mathrm{K}$ emission series characteristic of low atomic number elements including carbon, oxygen, nitrogen, etc., and the L emission series chosen for intermediate atomic number elements. The soft $\mathrm{x}$-ray emissions result from radiative transitions involving valence electrons. Consequently, the shape and the position of the maximum of soft emission bands are complex and depend on the electronic structure of the element within the matrix.

In the soft $\mathrm{x}$-ray region, the peak height is no longer proportional to the peak area and several approaches have been proposed to determine the intensity of a soft $\mathrm{x}$-ray emission band. For example, the use of predetermined "peak-to-area factor" as discussed by Bastin and Heijligers [5,6] or by measuring the peak area by summing the number of counts in each channel analyzed by stepping the monochromator across the wavelength domain containing the analyzed emission bands. The merits and limitations of these procedures have been discussed by Fialin et al. [7] accounting for the dependence of the peak shape on the self-absorption effect, the peak overlaps, and the resolution and the detection efficiency of the monochromator. However, it is still unclear whether the total area or only some spectroscopic features present in the measured spectra must be used to determine the intensity of the analyzed emission.

It is the aim of this paper to review the different features that lead to the complex shape of an x-ray line particularly within the soft $\mathrm{x}$-ray emission domain. Practical considerations for WDS spectra processing using least-squares fitting techniques will be discussed. Applications to the interpretation of WDS spectra to the study of the chemical environment and quantitative microanalysis using soft $\mathrm{x}$-ray emission bands will be illustrated using the $\mathrm{Fe} \mathrm{L} \alpha, \beta$ emission bands measured from pure iron and iron oxides.

\section{Contributions of X-Ray Generation Mechanisms and of Instrumental Factors to the Shape of a WDS X-Ray Line}

\subsection{The Diagram Lines}

The energy loss due to inelastic scattering events produces a hole in the inner-shell of an ionized atom. The de-excitation processes leads to the emission of a mono-energetic photon which is characteristic of the atom. The energy of the emitted photon is equal to the energy difference $\Delta E$ of the energy levels involved in the radiative transition (in a non-radiative transition, the excess of energy $\Delta E$ contributes to the emission of an Auger electron).

The emitted photon is characterized by a Lorentzian energy distribution with a width at half maximum $\Gamma$ (natural or physical width) related to the life time, $\tau$, of the hole on the initial state according to:

$$
\Gamma \tau=h / 2 \pi
$$

The natural profile of a radiative transition is a convolution of energy distributions of each of the levels involved in the transition, which is a Lorentzian curve whose FWHM is equal to the sum of the FWHM of the 
two levels. Broadening may occur with low energy levels if non-radiative transitions are possible such as Coster-Kronig transitions. The natural profile for transitions involving valence electrons are also broader than those resulting only from core holes.

The probability $P_{\text {if }}$ for a radiative transition between two levels $\mathrm{i}$ and $\mathrm{f}$ can be expressed according to:

$$
P_{\mathrm{if}}=\omega_{\mathrm{i}} z_{i f} N_{i}
$$

where $\omega_{\mathrm{i}}$ is the fluorescence yield, $z_{i f}$ is the weight of the line and $N_{\mathrm{i}}$ is the number of atoms in the initial level $\mathrm{i}$ per unit volume.

The fluorescence yield $\omega$ expresses the probability that the atom de-excites according to a radiative transition with the production of an x-ray photon. The probability to have a non-radiative transition with the emission of an Auger electron is (1- $\omega)$.

The fluorescence yield $\omega_{\mathrm{j}}$ of the level $\mathrm{j}$ is

$$
\omega_{\mathrm{j}}=N_{\mathrm{R}} /\left(N_{\mathrm{R}}+N_{\mathrm{NR}}\right)
$$

where $\mathrm{N}_{R}$ and $\mathrm{N}_{\mathrm{NR}}$ are the radiative and non-radiative transition rates, respectively.

For $n_{\mathrm{j}}$ ionisations created on level $\mathrm{j}$, the number of photons in the $\mathrm{j}$ series is $\left(\omega_{j} \cdot n_{j}\right)$.

The intensity $I_{\text {if }}$ in the radiative transition is proportional to $P_{\text {if }}$ [Eq. 6)] and depends on the convolution of the initial $D_{\mathrm{i}}$ and final $D_{\mathrm{j}}$ energy level distributions:

$$
I_{\text {if }}=P_{\text {if }}\left(D_{\mathrm{i}} * D_{\mathrm{j}}\right)
$$

For atomic lines involving only core levels, the convolution product $D_{\mathrm{i}} * D_{\mathrm{j}}$ is assumed to be a constant. However, this approximation is no longer valid with soft (low energy) $x$-ray emissions since the final states are valence hole states so that the emission spectra will change with the electronic structure (density of occupied states, DOS) of the material. The position of the maximum energy and the intensity of the emission band will vary as a function of the chemical environment.

In wide band gap materials such as aluminum oxide, the major peak of a soft $\mathrm{x}$-ray emission band (DOS) is usually accompanied by a low energy peak ("bonding peak") resulting from transitions to the initial hole of electrons from mixing $\mathrm{Al}_{3 \mathrm{sp}}$ and $\mathrm{O}_{2 \mathrm{p}}$ energy states in the valence band, as illustrated in Fig. 1. The bonding peak resulting from the mixing of states (referred as $\mathrm{K} \alpha{ }^{\prime}$ in the literature) is located at approximately $-5 \mathrm{eV}$ from the maximum of the $\mathrm{OK} \alpha$ parent peak. The feature labeled $\mathrm{K} \alpha$ " in Fig. 1 occurring on the short wavelength (high energy) side of the diagram peak may result from satellite emissions (see Sec. 2.2) or from instrumental effects, as discussed below.

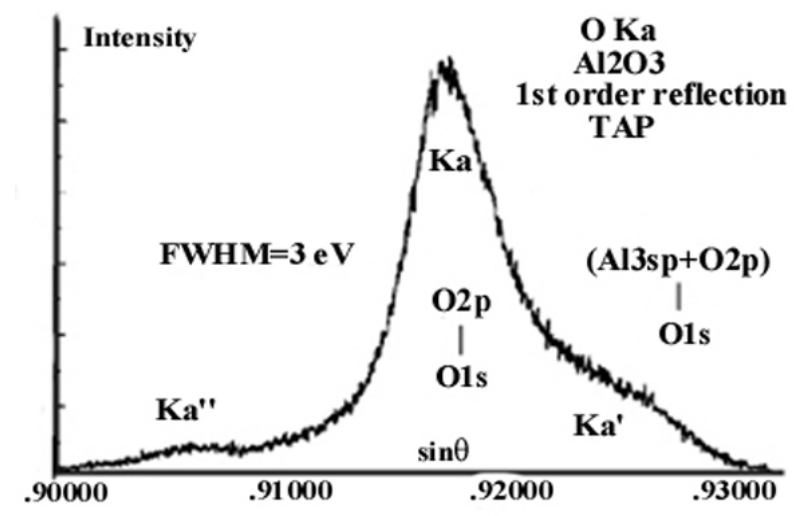

Fig. 1. OK $\alpha$ emission band measured with a TAP monochromator from an alumina crystal consisting from the diagram peak associated with $\mathrm{O}_{2 p}-\mathrm{O}_{1 s}$ transitions, a bonding peak associated with $\mathrm{Al}_{3 s p}, \mathrm{O}_{2 p}-\mathrm{O}_{1 s}$ transitions and a $\mathrm{Ka}^{\prime \prime}$ high energy satellite associated either with multiple ionizations or instrumental distortion.

In wide band gap crystals, some high energy features may also result from transitions involving levels located in the band gap of the energy diagram of the crystal. These levels are associated with intrinsic point defects which are induced either during the crystal growth conditions or induced during the specimen preparation (polishing with abrasives) or by radiolysis mechanisms during the electron irradiation. In oxides, the most frequent defects are $\mathrm{F}^{+}$and $\mathrm{F}$ centers, i.e., oxygen vacancies with one or two trapped electrons, respectively. As an exemple, Jonnard et al. [8] showed that the AlK $\beta$ emission $(3 p-1 s$ transition) from alumina crystals is accompanied by a small high energy weak emission peak located $0.6 \mathrm{eV}$ above the top of the valence band.

\subsection{The Non-Diagram Lines}

An x-ray emission line (or diagram line) resulting from a transition between two levels in the energy-level diagram is frequently accompanied by satellites (or non-diagram lines), i.e., x-ray lines whose energies do not correspond to the difference of two energy levels.

\subsubsection{High Energy Satellites}

The high energy satellite lines have been intensively studied since the 1930s to 1940s beginning with the detailed works of Parratt $[9,10]$ and Randall and Parratt [11]. Satellite lines result from electronic rearrangement concomitant with the ionization process during the de-excitation mechanisms of the ionized atoms.

$K$ Lines: When $1 s$ and $2 p$ vacancies are created simultaneously, the $2 p$ vacancy has a relatively long life-time compared to that of the $1 s$ vacancy. Thus, the 
inner vacancy de-excites in presence of a spectator hole which produces a change in the electrostatic potential leading to shifts in the energy levels (Fig. 2). The energy shifts for the $\mathrm{K} \alpha$ lines are given by:

$$
\Delta E=(\Delta E)_{1 s}-(\Delta E)_{2 p}
$$

The satellite lines resulting from the presence of outer vacancies consist of a number of closely spaced features. For the case of the $\mathrm{K} \alpha$ emission line, the high energy satellites are usually labeled as $\mathrm{K} \alpha_{3,4}$. The high energy satellite resulting from the de-excitation in presence of two outer vacancies is referred as $K \alpha_{5,6}$ and exhibits a very weak amplitude. The energy separation distance between the satellite band and the $\mathrm{K} \alpha$ line ranges from about $10 \mathrm{eV}$ up to about $40 \mathrm{eV}$ for atomic number $12<Z<30$. Aberg [12] presents an extensive set of values for the relative intensity of the satellite which decreases from about $30 \%$ for $Z=10$ to $0.5 \%$ at $Z=30$.

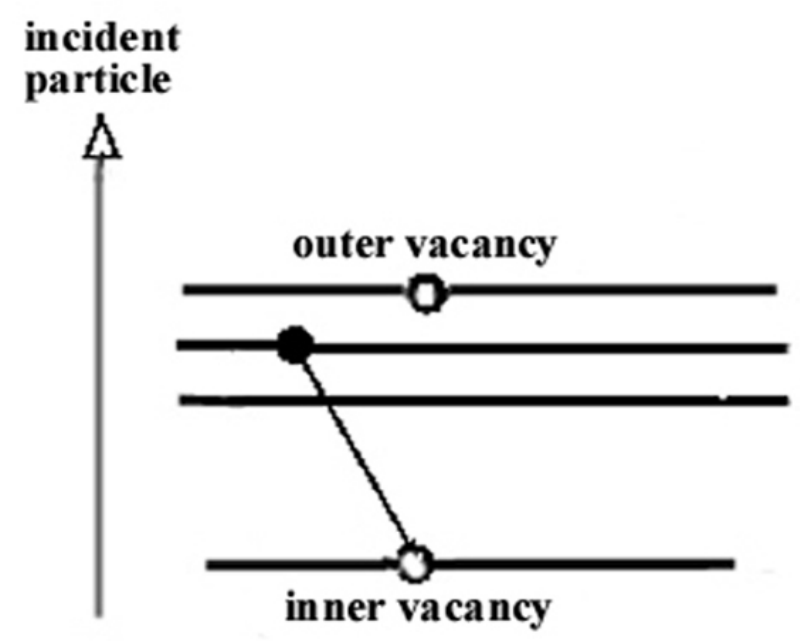

Fig. 2. Emission of $K \alpha_{3,4}$ high energy satellites.

$L$ and $M$ Lines: The de-excitation of an $\mathrm{L}$ or $\mathrm{M}$ level in presence of outer holes may also lead to the presence of high energy satellites associated with L or M X-ray peaks. The additional outer vacancies may result from Coster-Kronig transitions or shake-off mechanisms. The Coster-Kronig transitions result from an Auger process between sub-shells of the same shell.

The hole created on the $\mathrm{L}_{1}$ sub-shell may be filled by an electron originating from the $\mathrm{L}_{2}$ or $\mathrm{L}_{3}$ subshell. According to the selection rules, these transitions are not radiative and the excess of energy $\mathrm{L}_{1}-\mathrm{L}_{2}, \mathrm{~L}_{2}-\mathrm{L}_{3}$ or $\mathrm{L}_{1}-\mathrm{L}_{3}$ is dissipated by the emission of an Auger electron from the $\mathrm{M}$ or $\mathrm{N}$ levels. The transition rate of non-radiative Coster-Kronig transitions $f_{\mathrm{ij}}$, where $\mathrm{i}$ and $\mathrm{j}$ are two subshells within the same energy level, is not permitted for all elements.
Indirect ionizations resulting from the non-radiative Coster-Kronig process have the following effects on the emission profile:

1) To create additional vacancies so that the total number of ionizations is the sum of the direct ionizations produced by the incident electrons and those created by the non-radiative Coster-Kronig transition. For example the $\mathrm{L} \alpha$ emission line involving ionization on the $\mathrm{L}_{3}$ subshell, the number of $\mathrm{L} \alpha$ photons will be:

$$
n_{\mathrm{L}_{3}}<\omega_{\mathrm{L}_{3}}>=\omega_{\mathrm{L}_{3}}\left[n_{\mathrm{L}_{3}}+f_{13} n_{\mathrm{L}_{1}}+f_{23}\left(n_{\mathrm{L}_{2}}+f_{12} n_{\mathrm{L}_{1}}\right)\right]
$$

where $f_{13}, f_{23}$, and $f_{12}$ are the Coster-Kronig transition probabilities.

2) To leave outer vacancies during the re-arrangement of the ionized states between the sub-shells prior to the radiative transition with the emission of an x-ray photon. This process is responsible for the production of high energy satellites. When the atomic number of the emitter decreases, the energy separation distance between the shake-off satellites and the diagram also decreases and may be observed as a shoulder to the major peak.

According to Fabian [13], several line shapes of $\mathrm{L}$ emission spectra of elements in the first transition series can be distinguished depending on the incident electron energy region: 1) The Threshold Excitation Region when the incident energy lies between the $\mathrm{L}_{3}$ and $\mathrm{L}_{2}$ energy thresholds, multiple vacancy satellites are largely reduced, 2) The Satellite Region in which the L $\alpha$ diagram line becomes distorted by the progressive development of high energy satellites when the incident electron energy increases from the $\mathrm{L}_{3}$ sub-shell threshold up to about three times that value, and 3) The Self Absorption Region for incident energies greater than about 3 or 4 times the $L_{3}$ threshold energy, the fine structure vanishes and the effect is attributed to selfabsorption. Increasing incident electron energy also increases the absorption path of the generated x-ray photons within the specimen and self-absorption removes the fine structure when a high incident energy is used.

Peak shape changes as a function of the incident energy is illustrated in Fig. 3 for the case of the CuL $\alpha$ emission from pure copper measured with a TAP monochromator. The variation of the intensity of the high energy satellite relative to that of the diagram peak as a function of the beam energy results from a differential self absorption effect because the $\mathrm{L}_{3}$ absorption edge occurs between the two spectral features.

Similarly, high energy satellites associated with $\mathrm{M} \alpha$ lines of elements with high atomic number result from the $\mathrm{M}_{5}$ hole de-excitation in presence of simultaneous vacancies in the $\mathrm{M}_{5}$ and $\mathrm{N}$ sub-shells. Only the envelope 
of satellites resulting from additional vacancies in the $\mathrm{M}_{5}$ sub-shell can be distinguished from the diagram line.

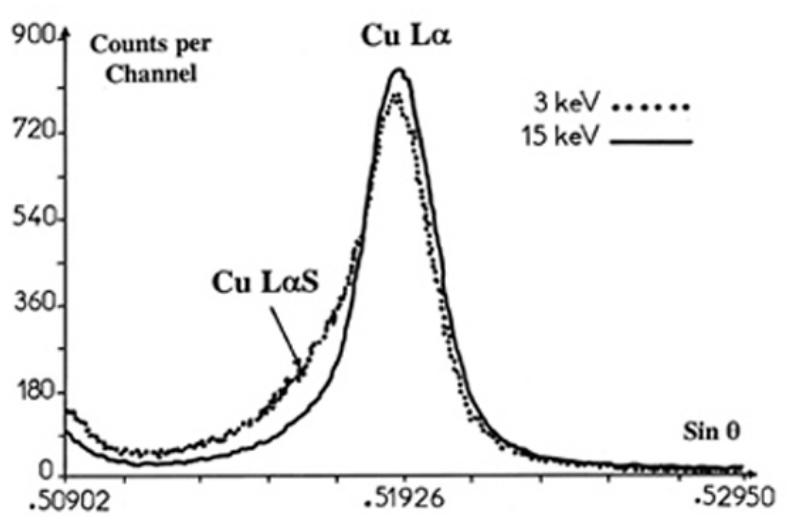

Fig. 3. $\mathrm{CuL} \alpha$ line shape changes as a function of the incident energy. Note that the $\mathrm{L}_{3}$ absorption edge lies between the main diagram peak and the high energy satellite.

As shown in Fig. 4, peak shape changes as a function of the beam energy are also observed for the case of the AuM $\alpha$ emission band measured from a pure $\mathrm{Au}$ specimen with a PET monochromator at $3 \mathrm{keV}$ and $15 \mathrm{keV}$ successively. The excitation energy thresholds for the $M_{1}$ to $M_{5}$ sub-shells are $3.425 \mathrm{keV}\left(M_{1}\right)$, $3.150 \mathrm{keV}\left(\mathrm{M}_{2}\right), 2.743 \mathrm{keV}\left(\mathrm{M}_{3}\right), 2.291 \mathrm{keV}\left(\mathrm{M}_{4}\right)$ and $20.206 \mathrm{keV}\left(\mathrm{M}_{5}\right)$, respectively. Thus, a $3 \mathrm{keV}$ incident energy is sufficient to provoke the $\mathrm{Au} \mathrm{M} \alpha$ emission involving the initial hole in the $\mathrm{M}_{5}$ sub-shell. Additional vacancies may be created in the $\mathrm{M}_{3}$ and $\mathrm{M}_{4}$ sub-shells with possible outer $\mathrm{N}$ vacancies resulting from CosterKronig transfer of the type $\mathrm{M}_{3,4}-\mathrm{M}_{5} \mathrm{~N}_{\mathrm{x}}$, producing weak high energy satellites. No additional vacancies are created in the $\mathrm{M}_{1}$ and $\mathrm{M}_{2}$ levels and transfer of the type $\mathrm{M}_{1,2}-\mathrm{M}_{5} \mathrm{~N}_{\mathrm{x}}$ does not exist for a $3 \mathrm{keV}$ incident energy.

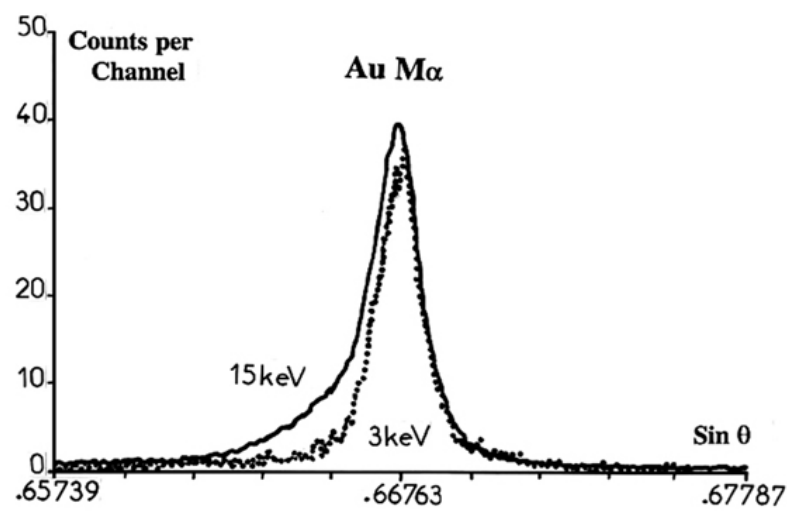

Fig. 4. Line shape changes of the AuM $\alpha$ emission peak as a function of the incident energy. The $3 \mathrm{keV}$ incident energy is lower than the $\mathrm{M}_{1}$ and $\mathrm{M}_{2}$ ionization thresholds.
Reciprocally, the $\mathbf{M}_{1}$ and $\mathbf{M}_{2}$ sub-shells are excited with a $15 \mathrm{keV}$ incident energy and the resulting inner vacancies can move to the $\mathrm{M}_{5}$ sub-shell with production of outer holes by Coster-Kronig mechanisms, thus the pronounced asymmetry on the high energy side of the $\mathrm{Au} \mathrm{M} \alpha$ may reasonably be assigned to the development of satellites resulting from the de-excitation of the $\mathrm{M}_{5}$ level in presence of outer $\mathrm{N}$ vacancies.

\subsubsection{Low Energy Satellites}

Several theories are available to describe the $\mathrm{K} \beta$ ' low energy feature associated with the $\mathrm{K} \beta_{1,3}$ emission resulting from transitions involving the partially filled $3 \mathrm{~d}$ shells of transition elements and their oxides.

The Radiative Auger Effect (RAE) produces a broad structure at a lower energy than the characteristic diagram line. The RAE process results from a deexcitation of a $\mathrm{K}$ vacancy, similar to an Auger process with simultaneous emission of a bound electron and an $\mathrm{x}$-ray photon (Fig. 5). For atomic number $15<Z<30$, the low energy structures associated with the $K \beta_{1,3}$ diagram line, can be interpreted in terms of KMM radiative Auger effect Radiative Auger Emission [14].

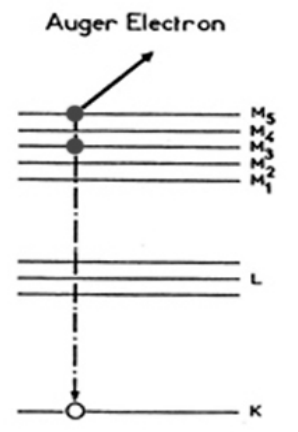
KMM Radiative Auger Emission: Simultaneous emission of a photon and an electron

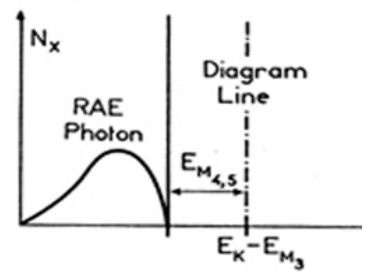

Fig. 5. Low energy satellite resulting from Radiative Auger Emission.

According to Salem et al. [15], the interaction between the electrons in the incomplete $3 \mathrm{~d}$ shell and the hole in the incomplete $3 p$ shell splits both $3 p$ and $3 d$ levels causing a demultiplication of transitions.

The $\mathrm{K} \beta$ ' satellite has also been explained in terms of the plasmon oscillation theory [16]. During the x-ray emission process, the transition valence electron excites a plasmon in the valence band. The transition energy of the $\mathrm{K} \beta_{1,3}$ line will thus be shared between the plasmon and the emitting photon which will be deprived of an energy equal to the plasmon energy. For the transition elements the energy separation distance between the $\mathrm{K} \beta^{\prime}$ satellite and $\mathrm{K} \beta_{1,3}$ diagram line is in the order of magnitude of $10 \mathrm{eV}$, depending upon the chemical environment [16]. 
The theories concerning the production of $\mathrm{K} \beta^{\prime}$ satellite associated with the $K \beta_{1,3}$ line of transition elements were extended to the case of the $\mathrm{L} x$-ray spectra of the lanthanide elements [17]. The $\mathrm{L} \beta_{2}, \mathrm{~L} \beta_{4}$, $\mathrm{L} \gamma_{1}$ and $\mathrm{L} \gamma_{2}$ emissions exhibiting low energy effects are associated with transitions involving the partially filled $4 f$ shell. The energy separation distance between the low energy satellite and its parent line is a few tens of $\mathrm{eV}$ and are easily detected with the resolution of the WDS of the EPMA as illustrated in Fig. 6, for the low energy (long wavelength) satellite labeled $\mathrm{L} \gamma_{10}$ associated to the HoL $\gamma_{2,3}$ peaks measured with a fully focusing quartz monochromator (Johannson mounting).

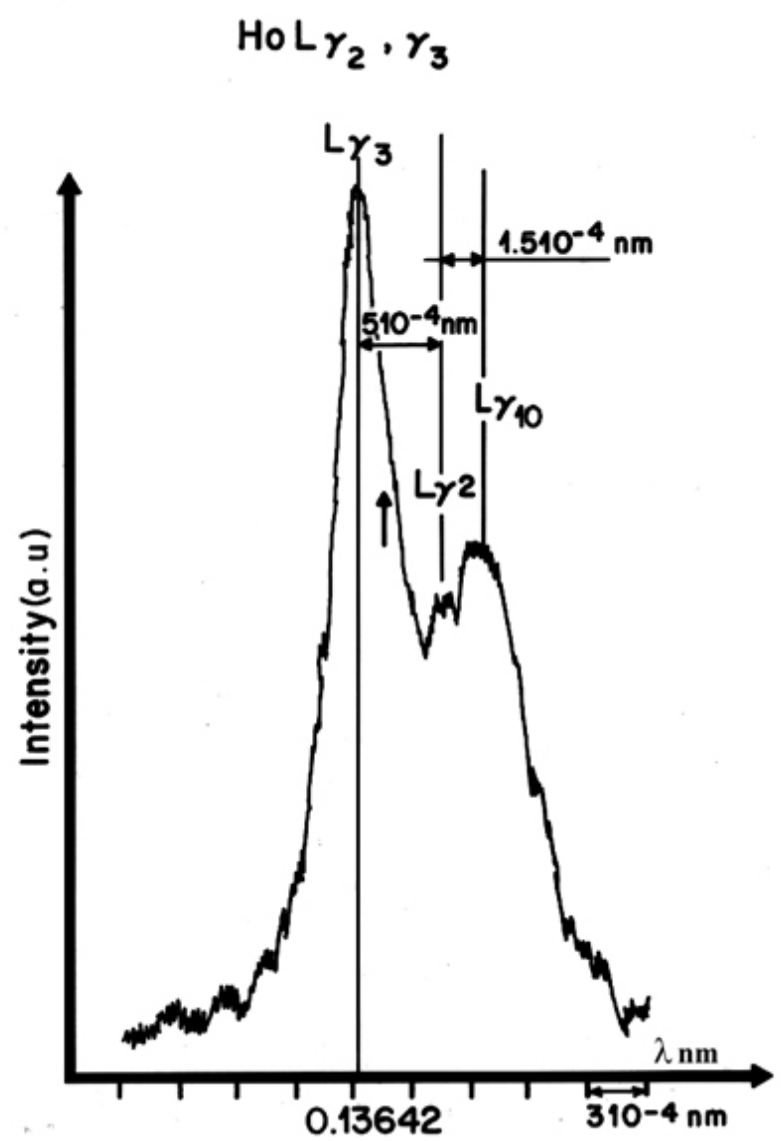

Fig. 6. Low energy satellite $\operatorname{HoL} \gamma_{10}$ associated with the $\operatorname{HoL} \gamma_{2,3}$ emission lines measured with a Johansson mounted quartz monochromator.

In practice, only the convolution of these features with the spectral window (or energy response function) of the spectrometer will be seen. Thus the ability to observe the non-diagram satellite bands will depend on the resolution and sensitivity of the spectrometer as reported by Rémond et al. [4,18] and Fialin et al. [2,3] for x-ray emission spectra measured with EPMA's equipped with WDS.

\subsection{Instrumental Distortions}

Modern EPMAs are generally equipped with no-slit spectrometers in which the monochromator is a crystal bent to yield a concave cylindrical surface producing a point focus image of the point $\mathrm{x}$-ray point source. In a symmetrical system, the curved Bragg planes are parallel to the crystal surface and the incident and "reflected" rays are located on the same side of the monochromator surface.

According to the Johann mounting, a flat crystal is cylindrically bent to twice the focal circle radius so that the focusing conditions are only satisfied for $\mathrm{x}$-ray beams incident at the "center" of the monochromator, i.e., the point where the focal circle is tangent to the crystal surface. A deviation from the focusing conditions will increase the further the incident $\mathrm{x}$-ray beam is from the center of the monochromator (semifocusing geometry). Away from the center of the crystal the small distance between its surface and the focal circle will give rise to a focusing defect, producing a broadening and a decrease in intensity of the observed x-ray peak According to Cauchois and Bonnelle [19], the line width due to departure from the Bragg conditions in the median plane of the crystal is given by:

$$
\Delta L=(\vartheta 2 / 8 R) \cot \theta
$$

where $\vartheta$ is the linear opening of the crystal and $R$ the focal circle radius.

Reducing the linear opening will minimize the focusing defect resulting from departure from point-topoint focusing conditions (point source and point image being both located on the focal circle) when a Johann mounted bent crystal is used as shown in Fig. 7 for the $\mathrm{Au} \mathrm{L} \alpha$ emission line measured with a Johann mounted $\mathrm{LiF}$ monochromator. The measurements were successively performed using the full area of the monochromator and after its active area was reduced by covering the edges of the crystal with narrow bands of lead. (Rémond et al. [18]).

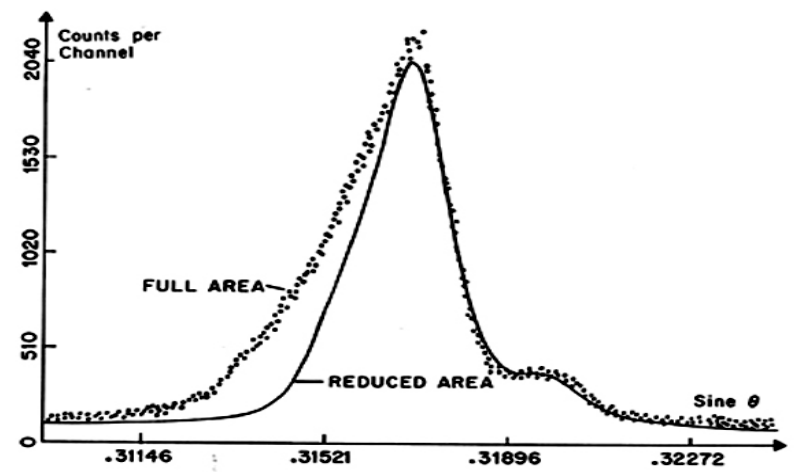

Fig. 7. Line shape of the AuL $\alpha$ peak analyzed using the full area of a $\mathrm{LiF}$ monochromator and after reducing the active area of the same monochromator. 
The line broadening for rays far from the center of the crystal cancels for a curved crystal of the Johansson type, i.e., when the Bragg focusing conditions are satisfied for all x-rays impinging the crystal surface. According to this mounting set-up, the crystal planes are bent to a radius of curvature $2 R$ and the surface of the crystal is ground to a circle radius $R$. With this geometry, the entire surface of the crystal is tangent to the focusing circle and the Bragg conditions are satisfied for all points at the monochromator surface (fully focus ing geometry).

The observed peak profile and intensity depend on the reflectance coefficient, $R(\theta)$, of the crystal for the direction $\theta$ with :

$$
R(\theta)=I_{\mathrm{R}} / I_{0}
$$

where $I_{\mathrm{R}}$ and $I_{0}$ are the intensities of the reflected and incident $\mathrm{x}$-ray beams, respectively. The graph of $R(\theta)$ as a function of $\theta$ is the diffraction pattern of the crystal. The reflecting power $P$, is given by

$$
P=\int R(\theta) \mathrm{d} \theta
$$

The reflectance coefficient is a function of the refractive index. For $x$-ray frequencies, the refractive index $\langle n\rangle$ is complex:

$$
<n>=n-\mathrm{i} \beta
$$

The real part of the refractive index, $n$, is slightly lower than unity and the decrement $\delta=1-n$ characterizes the dispersion. The imaginary part, $\beta$, of the refractive index is the extinction coefficient. It is related to the ordinary linear absorption coefficient $\mu$ by :

$$
\beta=(\lambda / 4 \pi) \mu
$$

The refractive index is complex, the extinction coefficient introduces a decrease of the amplitude of the waves passing through the crystal and phase changes between the incident and successively reflected waves

Neglecting the absorption due to the photoelectric effect and incoherent scattering, Darwin [20] showed that the reflection from a perfect plane crystal should be total over a narrow angular range, $\Omega$, outside this range the reflection diminishes rapidly and symmetrically. However, the diffraction pattern of a perfect plane crystal, lies below the Darwin curve and has an asymmetrical shape due to absorption resulting from the complex nature of the refractive index, i.e., decrease of the amplitude and phase changes between the waves.
For a crystal bent with a long radius of curvature, the reflectance curve remains very similar to the Darwin band of a flat monochromator. For short radius of curvature, the angle of reflection will change appreciably as a function of depth below the crystal surface leading to an exponentially decreasing tail on the low Bragg angle side.

Cauchois and Bonnelle [19] showed that the resulting Darwin curve for the bent crystal is made-up of adjacent rectangles of decreasing height as shown schematically in Fig. 8.

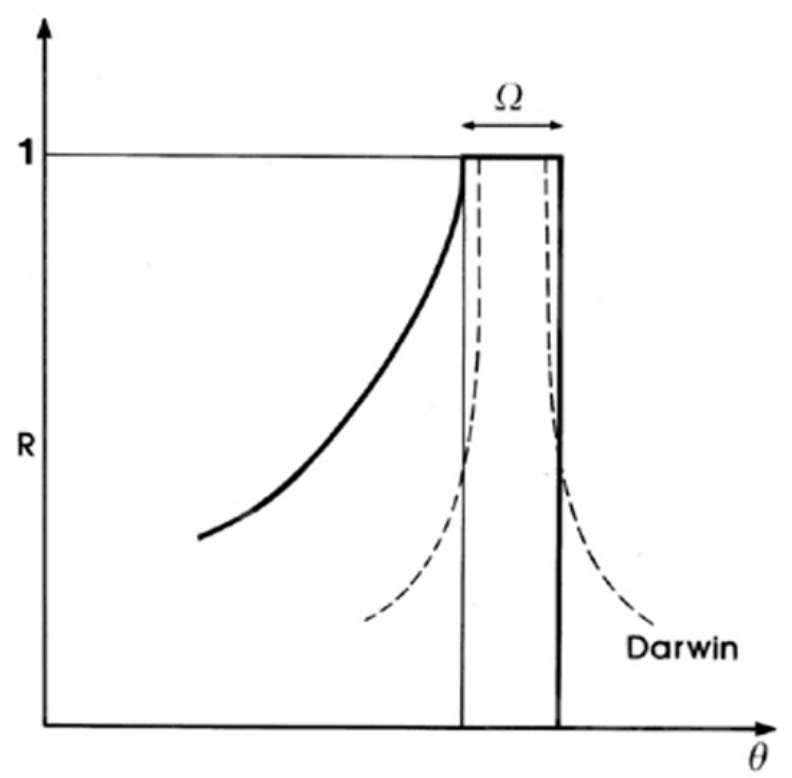

Fig. 8. Diffraction pattern of a bent monochromator.

The line width, $\Delta L$, arises from the intrinsic nature of multiple reflections inside the monochromator. According to Cauchois and Bonnelle, [19] the line width, $\Delta L$, along the focal circle, i.e., for rays incident near the center of the crystal so that the source and its image are on the same focal circle of radius $\mathrm{R}$ is:

$$
\Delta L=R \Omega+(\cos \theta / 2 \mu) \ln 2 .
$$

The first term, $R \Omega$, corresponds to a symmetrical contribution to the shape of the observed line and the second term, $(\cos \theta / 2 \mu) \ln 2$, expresses an asymmetrical tail occurring on the short wavelength side of the peak

At high Bragg angles $\left(\theta>>35^{\circ}\right)$ or for high absorption of the incident photons, only the outer surface of the crystal reflects the beam and the crystal behaves as a perfect crystal and second term in Eq. (12) is negligible. However, at low Bragg angles or for high energy rays penetrating deep in the crystal, this term is no longer negligible and an asymmetric diffraction pattern is found. 
The effect of the Bragg angle on the shape of an x-ray peak is illustrated in Figs. 9a,b for the first and second order reflection of the AuL $\alpha$ emission measured with a Johann mounted LiF monochromator.
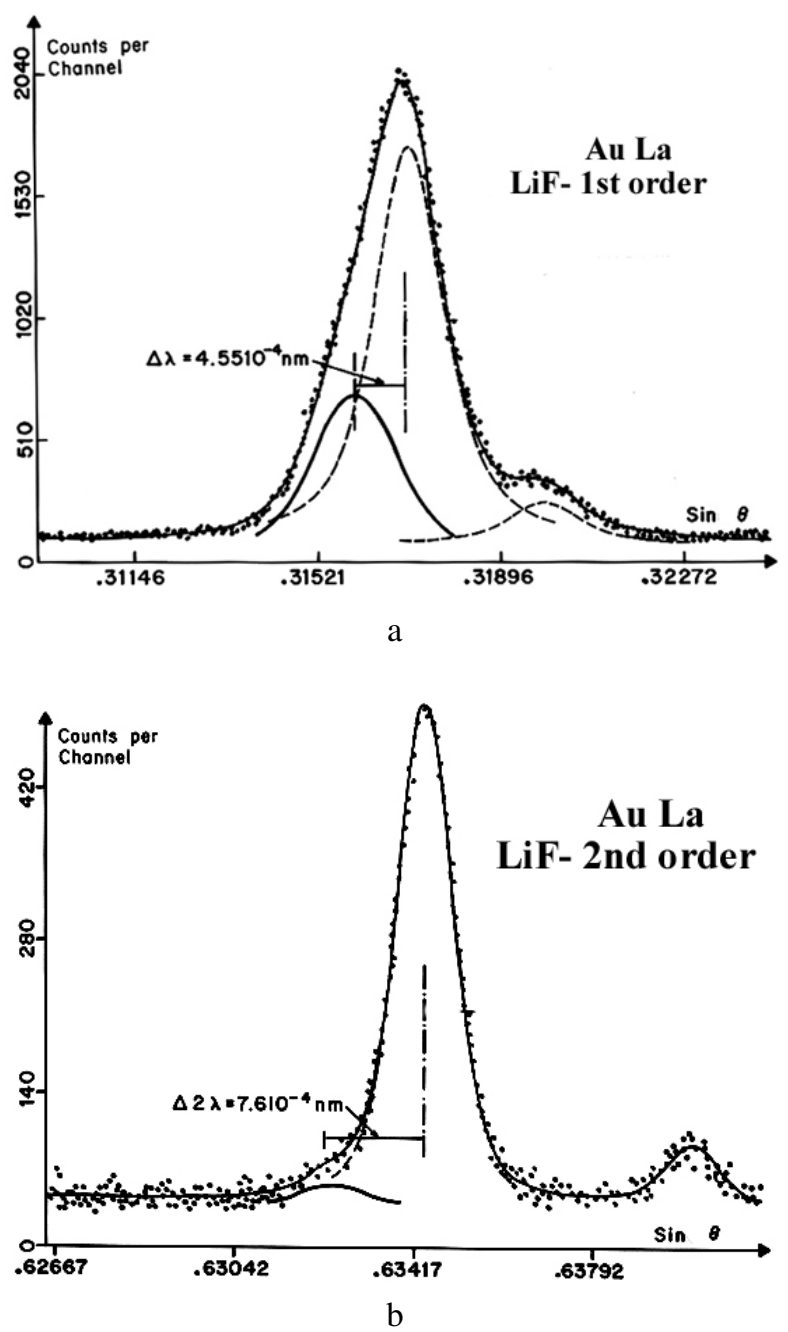

Fig. 9. Instrumental distortion of x-ray lines occurring at low Bragg angles. (a) first order reflection AuL $\alpha$ peak measured with a $\mathrm{LiF}$ monochromator (17 50" Bragg angle) and (b) second order reflection.

The broadening due to the thickness of the crystal will affect all incident rays impinging upon the surface along the focal circle and the $\Delta L$ broadening effect will occur either for Johann or Johansson curved crystals. The focusing defect resulting from the thickness of the crystal is illustrated in Fig. 10 for the AuL $\alpha$ peak measured with a quartz monochromator installed according to the Johansson mounting. For this measurement, the incident energy was set at $12.5 \mathrm{keV}$, a value just above the $\mathrm{L}_{3}$ excitation threshold but lower than that

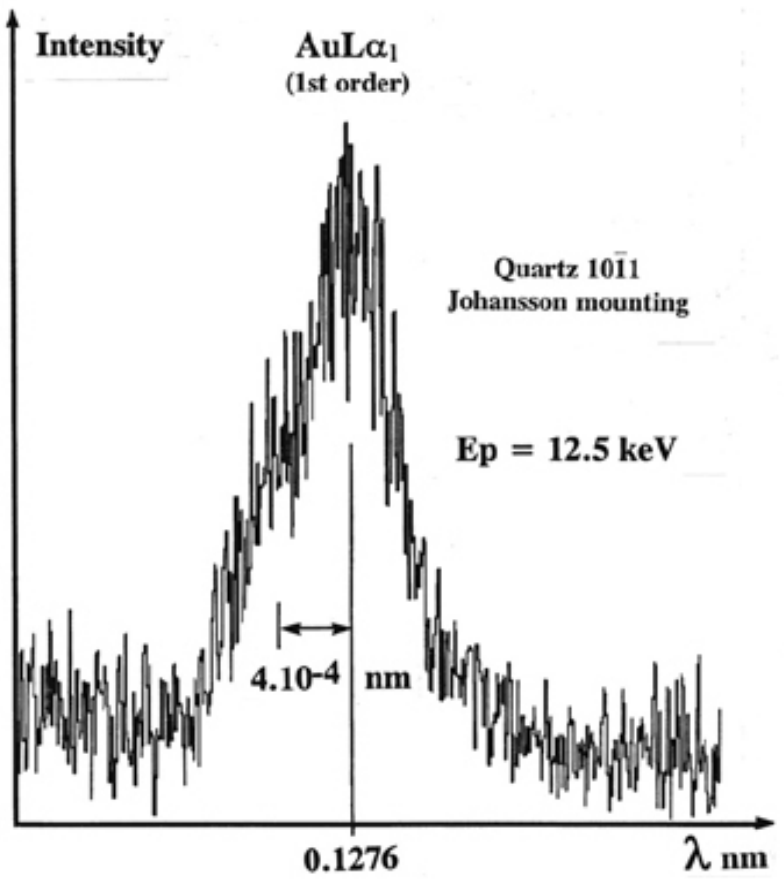

Fig. 10. First order reflection of the AuL $\alpha$ peak analyzed with a quartz monochromator (Johansson mounting) and a $12.5 \mathrm{keV}$ incident energy just above the $\mathrm{AuL}_{3}$ excitation threshold.

of the $\mathrm{L}_{1}$ and $\mathrm{L}_{2}$ levels. Under these conditions, the Coster-Kronig transitions are not produced and the tail observed on the short wavelength side of the AuL $\alpha$ peak must be assigned to an instrumental defect rather than a high energy satellite.

In some instances, artifacts may result fom the interactions of the incident $\mathrm{x}$-ray photons with the monochromator.

For example, it may be difficult to identify with certainty the $\mathrm{Ka}^{\prime \prime}$ band shown in Fig. 1 with the presence of a high energy satellite due to the $\mathrm{OK} \alpha$ emission resulting from de-excitation in presence of outer vacancies because an anomalous reflectivity of the monochromator containing oxygen may also lead to the presence of a parasite band. As reported by Mattson and Ehlert [21], the weight of the instrumental $\mathrm{Ka}^{\prime \prime}$ artifact to the OKa main peak is about $50 \%$ when measured with a KAP monochromator and is considerably reduced when measured with a TAP monochromator.

Another example of artifacts encountered in WDS is the presence of a "hole" in the continuous emission distribution as reported by Self et al. [22]. For a symmetrical reflection geometry using a bent monochromator, reflection of the $\mathrm{x}$-ray beams by crystallographic planes not parallel to the crystal surface may occur. In a single crystal, diffraction can occur from any atomic planes 
The monochromator is assumed to be bent along the $(h k l)$ planes. If an x-ray beam makes an angle, $\theta$, with the planes and an angle, $\theta^{\prime}$, with the $\left(h^{\prime} k^{\prime} l l^{\prime}\right)$ planes, the same wavelength will be diffracted by the two sets of planes when :

$$
2 d(h k l) \sin \theta=2 d^{\prime}\left(h^{\prime} k^{\prime} l^{\prime}\right) \sin \theta^{\prime} .
$$

In this situation, diffraction by the $\left(h^{\prime} k^{\prime} l^{\prime}\right)$ plane will cause a decrease in the intensity of the primary beam to be diffracted by the $(h k l)$ planes.

The presence of the "hole" in the intensity distribution of the continuous emission can be related to multiple reflections on planes differently orientated below the monochromator surface. Self et al. [22] calculated the positions of "holes" occurring at specific wavelengths in the continuous spectrum by considering diffraction from crystallographic planes different from the (200) planes of the LiF monochromator.

The simultaneous contribution of the hole in the continuous emission and the instrumental distortion due to the thickness of the crystal is illustrated in Fig. 11 for the AuL $\alpha$ peak characteristic of Au present at trace level in an arsenopyrite (AsFeS) specimen. This example clearly illustrates the need for spectral decomposition of the observed peak in order to derive accurate intensities as previously discussed by Rémond et al. [18,23].

\section{Deconvolution vs Spectral Decomposition Using Least-Square Fitting Techniques}

The observed photon distribution $P(E)$ within an $\mathrm{X}$-ray emission peak is expressed as:

$$
P(E)=\int L\left(E^{\prime}\right) F\left(E-E^{\prime}\right) \mathrm{d} E^{\prime}
$$

where $L(E)$ is the physical photon energy distribution, $F(E)$ is the instrumental response function, $E^{\prime}$ is the energy of the x-ray radiation and $E$ is the energy at the center of the peak.

Equation (14) can only be solved if the $L(E)$ and $F(E)$ distributions are known for all analysed photon energies. The natural width of an x-ray emission is generally well described according to a Lorentzian distribution

$$
L(E)=\frac{H}{1+\left[\left(E-E_{0}\right) / \gamma\right]^{2}}
$$

where $\gamma$ is the half-width at half maximum (HWHM), $\mathrm{H}$ is the amplitude of the distribution centered at energy $E_{0}$. The response function $F\left(E^{\prime}\right)$ expresses the observed line shape of a mono-energetic photon assumed to have a natural width equal to zero (or negligible) with respect to the energy resolution of the spectrometer.

In practice, for quantitative analysis with the EPMA, a full deconvolution procedure is not required and the data processing only aims to measure the intensities of partly or fully overlapping components in the observed spectrum. Instead of deconvolution procedure in the strict sense based on Eq. (24), spectral decomposition based on least square fitting techniques of the measured spectrum to analytical descriptions of $\mathrm{x}$-ray peaks is more frequently used in EDS and WDS quantitative $\mathrm{X}$-ray analysis. The fitting function describing the shape of the observed peak includes the peak position, the peak intensity and the peak width as variables. In an analytical description of the peak shape only an effective width representing the combination of the natural and instrumental contributions is used.
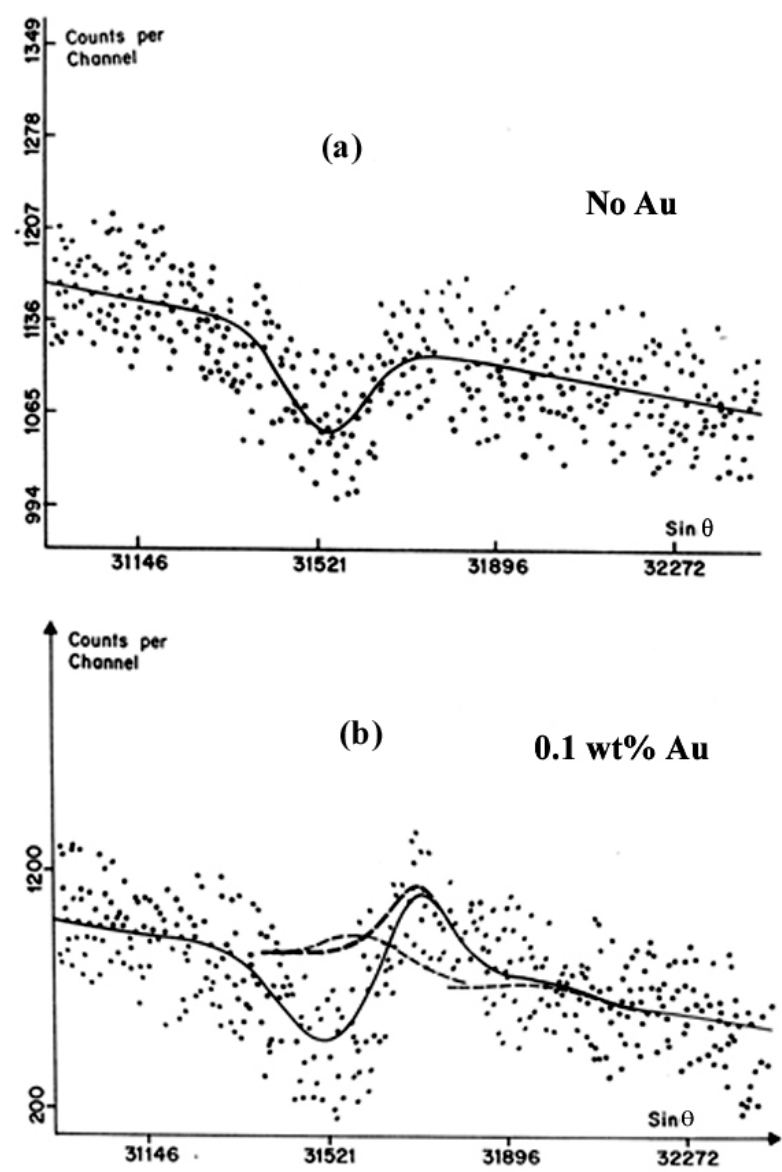

Fig. 11. Effect of instrumental distortions on the observed AuL $\alpha$ line analyzed with a $\mathrm{LiF}$ monochromator. (a) decrease of the continuous emission intensity resulting from multiple reflections on planes differently orientated below the surface monochromator, (b) observed AuL $\alpha$ peak for gold present at trace level resulting from the presence of the hole in the continuum emission and the instrumental distortion due to focusing defect of the monochromator as shown in Fig. 9a. 


\subsection{A Need for a Unique Approach for EDS and WDS Spectra Processing}

The energy resolution of an EDS spectrum obtained with a microcalorimeter [24] is similar to that obtained with a WDS as illustrated in Fig. 12 for the $\mathrm{OK} \alpha$ emission band from an $\mathrm{Al}_{2} \mathrm{O}_{3}$ specimen. Therefore, there is thus a need to use a unique analytical description of the shape of an X-ray line to least-squares fit to the WDS and high resolution EDS spectra.

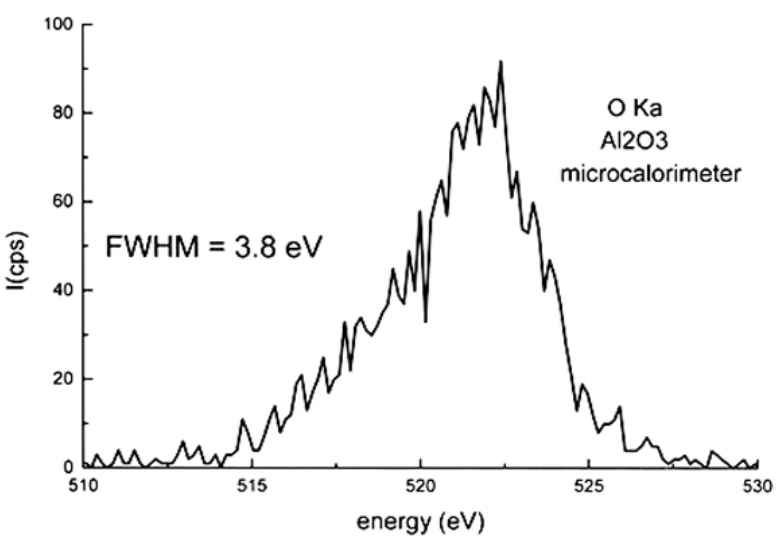

Fig. 12. $\mathrm{OK} \alpha$ from an alumina specimen measured with the microcalorimeter (spectrum measured by Dr. Wollman at NIST).

Studying the response of solid-state energy dispersive detectors to high energy mono-energetic incident radiations Phillips and Marlow [25] expressed the observed line shape $P(E)$ as a function of the analyzed photon energy $E$, according to:

$$
P(E)=S(E)+D(E)+G(E)
$$

The above expression is known as the Hypermet function in which $S(E)$ represents the Compton scattering of photons within the detector, $D(E)$ expresses the phenomena of incomplete charge collection in the dead layer of the solid-state detector and $G(E)$ is the major Gaussian peak whose the width is large with respect to the intrinsic width of the diagram line.

According to the Hypermet function, the asymmetry of peaks resulting from the photon-detector interactions is treated by adding two analytical expressions $S(E)$ and $D(E)$ to that describing the spectroscopic features. Several expressions for $S(E)$ and $D(E)$ are available depending upon the analyzed photon energy domain and the type of detectors as discussed by Campbell et al. [26].

It is widely accepted that describing a measured x-ray peak by a Gaussian distribution is a sufficient approximation to derive accurate $\mathrm{x}$-ray intensities from an $\mathrm{x}$-ray emission spectrum measured with an EDS. Most software available with commercial EDS systems use a
Gaussian approximation for describing the observed shape of an X-ray peak which is not distorted by instrumental factors. The incomplete charge collection phenomenon $D(E)$ in the dead layer of the detector, dominates the asymmetry of low energy $\mathrm{x}$-ray peaks analyzed by means of a $\mathrm{Si}(\mathrm{Li})$ detector.

\subsection{The Fitting Function to Mono-Energetic Features}

The observed shape of an x-ray line is controlled by the intrinsic properties of the spectrometer and its geometrical arrangement within the specimen chamber. Thus, depending on the resolution of the spectrometer, there is no evidence that the line shape function satisfies a purely Gaussian or a purely Lorentzian distribution function.

A pseudo-Voigt function, $P(\lambda)$, can be used as a fitting function to the measured WDS peak shape $[18,27]$ according to:

$$
P(\lambda)=C g G(\lambda)+1 L(\lambda)
$$

where $0 \leq C \mathrm{~g} \leq 1$ and $C \mathrm{l}=1-C \mathrm{~g}$ are the contributions of the Gaussian $G(E)$ and Lorentzian $L(E)$ of same width $(\Gamma \mathrm{g}=\Gamma \mathrm{l})$ and centered at the same position. Each function in the linear combination shown in Eq. (17) is weighted by a coefficient $C g$ expressing the intermediate nature between Gaussian and Lorentzian shaped WDS x-ray peaks.

\subsection{The Fitting Function to the Continuous Emission Distribution}

Spectral decomposition of either EDS or WDS characteristic $\mathrm{x}$-ray emission peaks can only be applied when the underlying continuous emission has been removed or when an analytical description of this emission has been added to the fitting procedure.

When a full WDS spectrum is measured it is necessary to account for the variations of the absorption as a function of wavelength as is the case when modeling the continuous emission for an EDS spectrum. For this purpose, a physical description of the WDS continuous emission should be used such as the model proposed by Smith and Reed [28] for the description of the continuous emission associated with WDS spectra of rare-earth bearing compounds.

When the absorption edge is located within the narrow wavelength domain containing the emission band, for example the $\mathrm{L}$ emission spectra of transition elements, it is necessary to correct the observed peak shape for self-absorption before extracting the peak intensities. 
According to Fabian [13], a self-absorption spectrum can be obtained from two spectra measured from two different excitation conditions. The method consists in normalizing the two spectra and then dividing them channel by channel.

The observed spectrum is the sum of the number of counts $I_{\mathrm{i}}$ in each channel $\mathrm{i}$ within the wavelength domain containing the line of interest. Let $\bar{I}_{\mathrm{i}}$, be the intensity $I_{\mathrm{i}}$ at channel $\mathrm{i}$, normalized to that $I_{0}$ at the peak maximum of the analyzed line.

Let us assume that the incident energy $E_{1}$ is low enough to neglect the absorption effect. Thus, $\bar{I}_{\mathrm{i}}\left(E_{1}\right)$ represents the generated intensity in channel $\mathrm{i}$ of a distribution whose the maximum intensity is equal to unity:

$$
\bar{I}_{\mathrm{i}}\left(E_{1}\right)=I_{\mathrm{i}}\left(E_{1}\right) / I_{0}\left(E_{1}\right)=I_{\mathrm{i}}\left(E_{1}\right) /\left[Z\left(E_{1}\right)\right]
$$

where $I_{0}\left(E_{1}\right)=\int \varphi(\rho z) \mathrm{d} \rho z=\left[Z\left(E_{1}\right)\right]$ is the atomic number correction factor since the absorption for $E_{1}$ is negligible.

For an incident energy $E_{2}>E_{1}$, the absorption effect is no longer negligible and $\bar{I}_{\mathrm{i}}\left(E_{2}\right)$ is the emitted intensity, in channel $\mathrm{i}$, of a distribution whose the maximum intensity is equal to unity expressed by:

$$
\left.\bar{I}_{\mathrm{i}}\left(E_{2}\right)=I_{\mathrm{i}}\left(E_{2}\right) / I_{0}\left(E_{2}\right)=I_{\mathrm{i}}\left(E_{2}\right) /\left[Z\left(E_{2}\right)\right] f\left(\chi_{0}\right)\right]
$$

since for the energy $E\left({ }_{2}\right)$ we have $I_{0}\left(E_{2}\right)=$ $\int \varphi(\rho z) \exp (-\chi \rho z) \mathrm{d} \rho z=\left[Z\left(E_{2}\right)\right] f\left(\chi_{0}\right)$, where $f\left(\chi_{0}\right)$ is the usual absorption correction factor for the wavelength corresponding to the peak maximum.

The intensity ratio between the two normalized spectra $\bar{I}_{\mathrm{i}}\left(E_{1}\right)$ and $\bar{I}_{\mathrm{i}}\left(E_{2}\right)$ measured at low $\left(E \mathrm{p} 2_{1}\right)$ and high $\left(E_{2}\right)$ incident energy successively is thus:

$$
g\left(\chi_{\mathrm{i}}\right)=\bar{I}_{\mathrm{i}}\left(E_{1}\right) / \bar{I}_{\mathrm{i}}\left(E_{2}\right)
$$

The graph $g\left(\chi_{\mathrm{i}}\right)$ of the ratio of the normalized intensities as a function of wavelength (or channel i) expresses the variations of the absorption correction factor relative to that at the peak maximum accounting for the presence of the absorption edges in the analyzed wavelength region as illustrated in Fig. 13 for the case of the $\mathrm{CuL} \alpha, \beta$ emission spectra.

Since the two normalized spectra have the same amplitude at their maximum, the intensities $\bar{I}_{\mathrm{i}}\left(E_{1}\right)$ and $\bar{I}_{\mathrm{i}}\left(E_{2}\right)$ represent the fraction of generated and emitted intensities respectively with respect to the maximum intensity equal to unity. Consequently, $g\left(\chi_{\mathrm{i}}\right)$ is an equivalent absorption correction factor in channel $\mathrm{i}$, relative to that for the channel corresponging to the peak maximum.

Combining Eqs. (18), (19) and (20) leads to:

$$
I_{\text {gen }}^{\mathrm{i}}=I_{\text {mea }}^{\mathrm{i}} * g\left(\chi_{\mathrm{i}}\right) * \frac{\left[Z\left(E_{1}\right)\right]}{\left[Z\left(E_{2}\right)\right] f\left(\chi_{0}\right)}
$$

where $I_{\text {gen }}^{\mathrm{i}}$ is the calculated generated intensity corresponding to the measured intensity $I^{\mathrm{i}}$ mea,$f\left(\chi_{0}\right)$ is the normal absorption correction factor for the wavelength corresponding to the peak maximum and [Z] designates the atomic correction factor. The graph $g\left(\chi_{\mathrm{i}}\right)$ is determined empirically from two measurements performed at low and high incident energy successively and the scaling factor $\frac{\left[Z\left(E_{1}\right)\right]}{\left[Z\left(E_{2}\right)\right] f\left(\chi_{0}\right)}$ is calculated using Monte-Carlo calculations or by means of the analytical expressions commonly used for quantitative $\mathrm{x}$-ray microanalysis.

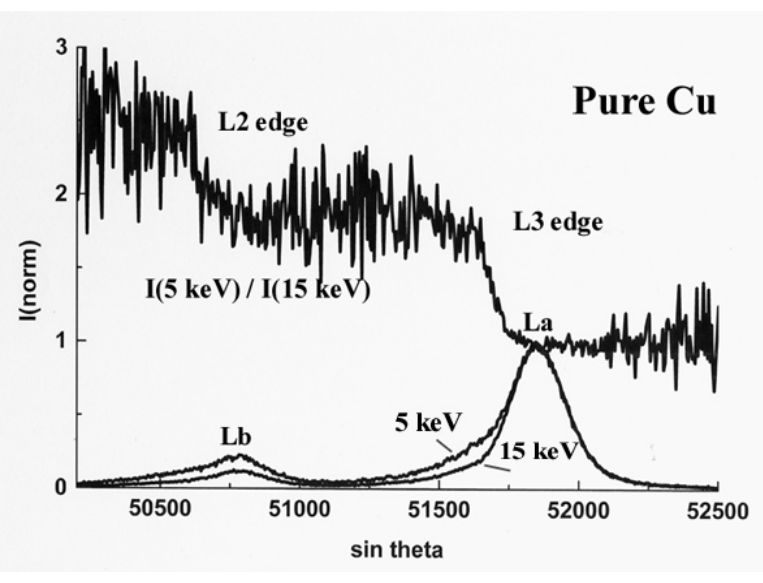

Fig. 13. Self-absorption correction factor for $\mathrm{CuL} \alpha$ in pure $\mathrm{Cu}$ derived from the intensity ratio of two $\mathrm{CuL} \alpha$ spectra measured at $3 \mathrm{keV}$ and $15 \mathrm{keV}$ incident energy successively.

A second approach for deriving the variation in the $f(\chi)$ correction factor within the analyzed wavelength domain containing an absorption edge has been previously discussed by Rémond et al. [5]. The approach consists in transferring the WDS spectrum into the energy space and to model the continuous emission $N(E)$ as a function of the energy according to:

$$
N(E)=Z\left[a\left(\frac{E_{0}-E}{E}\right)+b\left(\frac{E_{0}-E}{E}\right)^{2}\right] f(E) D(E)
$$

where $E_{0}$ is the incident energy, $E$ the current photon energy, $f(E)$ is the absorption correction factor at energy $E$ and $D(E)$ is the detection efficiency.

The continuous emission is calculated taking into account the presence of the absorption edges and then adjusted to the experimental spectrum. The calculation is repeated by omitting the presence of the absorption edges, i.e., assuming a continuous linear variation of the continuum in the analyzed energy domain. The ratio of the two calculated curves gives the correction factor to be applied to each channel as illustrated in Fig. 14 for 


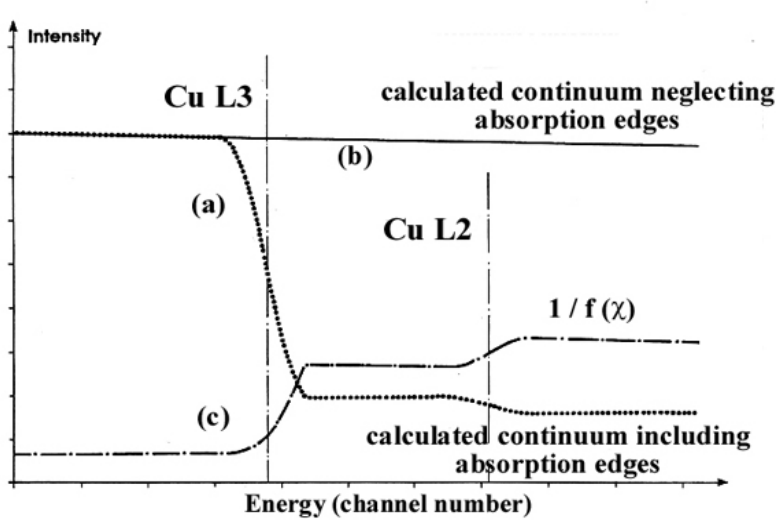

Fig. 14. Continuous emission distribution as a function of photon energies associated with the $\mathrm{Cu} L \alpha, \beta$ emission bands for a pure copper specimen. (a) calculation accounting for the absorption edges, (b) calculations neglecting the absorption edges, and (c) absorption correction factors given by the ratio of the two calculated (a) and (b) continuous emission distributions.

the $\mathrm{CuL} \alpha, \beta$ EDS spectrum derived from the experimental WDS data.

When the normalized absorption correction factors for each channel are obtained, it is possible to reconstruct the generated emission spectrum. Dividing the real value of each channel of an experimental spectrum measured with the high incident energy by the corresponding channel in the graph of the correction factor provides a spectrum whose the shape is corrected for the differential absorption within the analyzed wavelength domain. The corrected spectrum corresponds to an experimental spectrum superimposed on a constant linear continuum emission intensity within the wavelength domain containing the emission bands of interest. The generated emission spectrum is thus obtained by multiplying the content of each channel of the spectrum corrected for the presence of the absorption edges by the calculated value of the generated intensity at the peak maximum. That value is derived from the measured peak height intensity of the L $\alpha$ line using usual ZAF or $\varphi(\rho z)$ procedures with the absorption coefficient for the mono-energetic $\mathrm{L} \alpha$ line obtained from data tables.

\section{Applications of Least-Square Fitting Techniques to WDS Spectra}

\subsection{Practical Considerations}

The WDS x-ray peaks were digitally recorded by moving the monochromator step by step. Before displaying the $\mathrm{x}$-ray peaks, the measured intensity in each channel was corrected for dead time.

For each analyzed peak the fitting procedure was conducted using a set of pseudo-Voigt profile with additional Gaussian or pseudo-Voigt offsets and except when specified, the intensity of the continuous emission underlying the analyzed x-ray peaks was approximated by a linear function.

The adjustment of the fit of the experimental spectra to the model function is done with an interactive multiple least square fitting program developed by Massiot [29], minimizing the residual distance normalized to the number of data points (standard deviation $\mathrm{SD})$ according to

$$
S D=\left(\sum \frac{\left[P^{1}\left(\lambda_{\mathrm{i}}\right)-P^{1}\left(\lambda_{\mathrm{i}}\right)\right]^{2}}{n}\right)^{1 / 2}
$$

where $n$ is the number of data points, $P^{\prime}(\lambda \mathrm{i})$ is the number of counts at channel $i$ and $P^{\prime}(\lambda i)$ is the value of the model function at that point $i$. The method utilizes an iterative non-linear least-squares fitting process starting from an initial estimated solution.

The shape of a peak resulting from the simultaneous presence of a diagram and non-diagram bands can be described by the sum of analytical pseudo-Voigt functions which describe each of the spectral components.

In previous studies, Rémond et al. [4,18] added Gaussian or pseudo-Voigt (equation [17] offsets to the main profiles to describe the high energy tail of x-ray emission peaks occurring at low Bragg angles. This approach based on the addition of high energy offsets, is supported by the results by Cauchois and Bonnelle [19] who showed that the asymmetrical diffraction pattern for a bent monochromator can be approximated by a sum of adjacent rectangular Darwin curves with decreasing amplitudes.

An observed $\mathrm{x}$-ray peak will be described as the sum of many pseudo-Voigt profiles, each of them being characterized by four parameters, the proportion $C \mathrm{~g}$ of Gaussian to Lorentzian distribution, the peak position, the peak width and the peak amplitude. With the available least-squares fitting program used, the parameter $C \mathrm{~g}$ must be empirically determined and used as a constant in the fit, all other parameters being kept as variables. When possible, some of the variables must be predetermined and used as constants or be coupled to each other in order to impose physical constraints and to reduce the number of variables.

\subsection{Gaussian to Lorentian Proportion and Line Widths}

As previously illustrated in Ref. [18], the shapes of the $\mathrm{SK} \alpha \mathrm{NbL} \alpha$ and AuM $\alpha$ diagram peaks of similar energy were analyzed with the PET monochromator and were found to be very different. The proportion of Gaussian and Lorentzian distributions in the peak 
profile is a function of the intrinsic properties of the analyzed emission, i.e., of the energy sub-shells involved in the analyzed radiative transitions.

In practice, the value of the $C \mathrm{~g}$ parameter in Eq. (17) is determined by varying step by step the $C \mathrm{~g}$ value from 0 (pure Lorentzian) to 1 (pure Gaussian) until a satisfactorily description of the long wavelength side of the peak is obtained. This approach assumes the absence of low energy satellites such as RAE satellites, which usually have weak amplitudes. This approach is more difficult to apply to unresolved $\alpha_{1}-\alpha_{2}$ doublets or to soft $\mathrm{x}$-ray emission bands since the bonding peak occurs on the low energy side of the diagram band as previously illustrated in Fig. 1. In the presence of overlapping peaks a data base is required to couple the peak positions and amplitudes in order to accurately determine the low energy peak profile.

In order to reduce the number of variables in the fit, a solution includes the determination of a calibration curve for the peak widths as a function of the wavelength domain analyzed by each monochromator. Such calibration curves should be useful to generate synthetic reference spectra.

In practice, specific calibration curves of peak widths as a function of wavelength must be obtained for emission lines belonging to the $\mathrm{K}, \mathrm{L}$, or $\mathrm{M}$ series since these lines of similar energy do not only exhibit shape changes but also have different peak widths [4]. These variations account for the contribution of the natural width to the observed peak width. Rémond et al. $[1,18]$ used an approach where the observed width, used as a variable in the fitting function, was replaced by quadratic addition of the instrumental resolution $\Gamma_{\mathrm{i}}$ and natural width $\gamma$, according to:

$$
\Gamma=\left[\Gamma_{\mathrm{i}}^{2}+\gamma^{2}\right]^{1 / 2}
$$

The width of $L \alpha$ and $L \beta_{1}$ peaks of pure elements analyzed with a PET monochromator (Bragg angles greater than $\approx 30^{\circ}$ ) exhibited a linear variation as a function of the analyzed wavelengths as shown in Fig. 15. From all analyzed L x-rays peaks, the resolution $\Gamma_{\mathrm{i}}$ of the spectrometer equipped with a PET monochromator was calculated using Eq. (22). As shown in Fig. 15, a linear relationship was found to exist between the analyzed $\mathrm{L}$ emission peak and the instrumental resolution $\Gamma_{\mathrm{i}}$. This relation can be used to determine the width of any peak in the analyzed wavelength domain and can be used as a constant in the fitting procedure rather than a variable. The validity of the simplified approach in Eq. (22) was supported by comparing

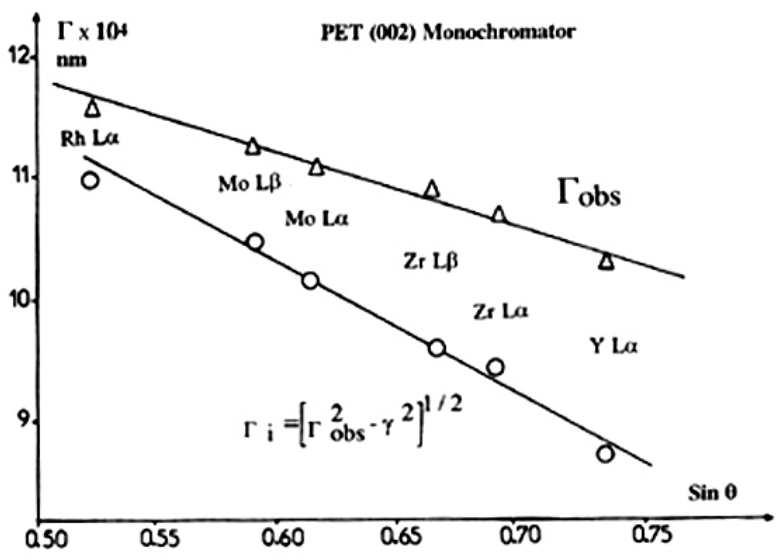

Fig. 15. Calculated FWHM and instrumental response for function for x-ray lines analyzed with a PET monochromator.

observed and calculated widths of the $\mathrm{NbL} \alpha(2196 \mathrm{eV})$ and $\mathrm{AuM} \alpha(2123 \mathrm{eV})$ lines measured with the PET monochromator [18].

The calculated width of the experimental $\mathrm{NbL} \alpha$ peak was found to be $4.1 \mathrm{eV}$, which differs by less than $3 \%$ of the observed peak width of $3.97 \mathrm{eV}$. Similarly, the calculated intrinsic width for the AuM $\alpha$ line was found to be $2.7 \mathrm{eV}$, which is very close to intrinsic width at $2.5 \mathrm{eV}$ reported by Laakkonen and Graeffe [30].

Differences in width not only exist between $\mathrm{K}, \mathrm{L}$, and $\mathrm{M}$ emission lines of similar energy, but also exist between the multiple lines of the $\mathrm{L}$ series of the same element. Neglecting the contribution of the natural width to the observed width may lead to the detection of artifacts as illustrated for $\operatorname{SmL} \beta_{3}$ emission line [1]. Peak shape analysis of the $\operatorname{SmL} \beta_{3}$ using the width as a variable in the fit leads to a larger width of the peak than that of the $\operatorname{SmL} \alpha$ peak. It was thus tempting to identify the $\mathrm{L} \beta_{3}$ peak broadening as the result of two unresolved lines as shown in Fig. 16a. For these calculations, the width of the two components were set at the value derived from the calibration curve $\Gamma_{\mathrm{i}}=f[\Gamma(\mathrm{L} \alpha)]$. Such decomposition led to the detection of a spurious peak since the $\mathrm{L} \beta_{3}$ is a single line as shown in Fig. 16b when the width of the peak was set to that calculated by Eq. (22), including the instrumental resolution of the monochromator and the natural width of the $\mathrm{L}_{3}$ line. As shown in Fig. 17, the natural widths of the $\mathrm{L} \beta_{3}$ lines for the rare-earth elements are approximately twice those of the $\mathrm{L} \alpha$ line of the same element [31]. These differences are responsible for the measured widths derived from the peak shape analysis of the $\mathrm{L}$ lines of the rare-earth elements when the width of each component is kept as a variable in the fitting procedure. 


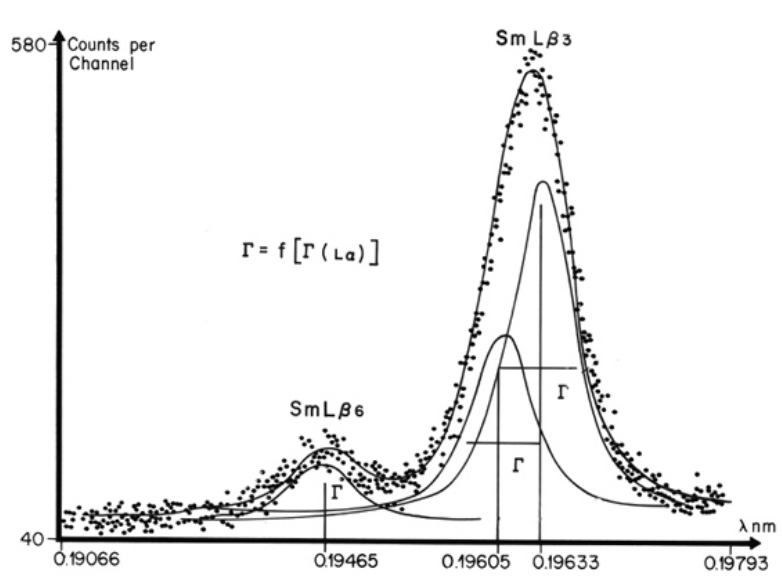

a

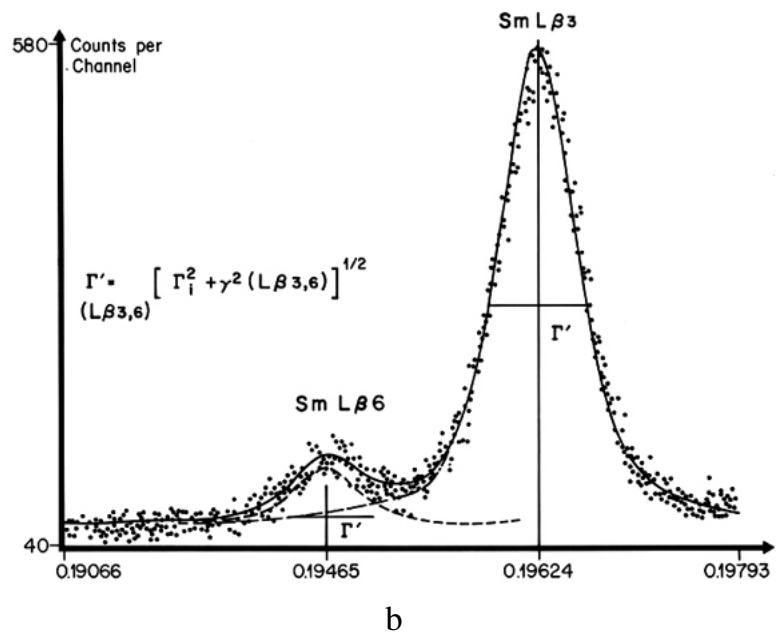

Fig. 16. Results of fits to the $\operatorname{SmL} \beta_{3,6}$ peaks analyzed with a $\mathrm{LiF}$ monochromator. (a) using the FWHM value derived from a calibration curve of widths for $L \alpha$ lines and (b) using FWHM derived from Eq. (22) accounting for the natural width and the instrumental resolution.

\subsection{Relative Intensities}

Data in Fig. 18 correspond to a $\mathrm{ZnO}$ matrix showing interference between the fundamental $\mathrm{OK} \alpha$ emission band and the second order reflection of the $\mathrm{ZnL} \alpha, \beta$ emission bands analyzed with a W/Si multilayer structure as monochromator.

The positions, the relative intensities $\mathrm{ZnL} \alpha, \beta$ and the intensities of the high energy satellites to the $\mathrm{L} \alpha$ and $\mathrm{L} \beta$ lines depend on the chemical environment and these parameters must be determined and used as coupled variables in the fit in order to obtain an accurate intensity of the $\mathrm{OK} \alpha$ band which is interfering with the $\mathrm{ZnL} \alpha, \beta$ second order reflection.

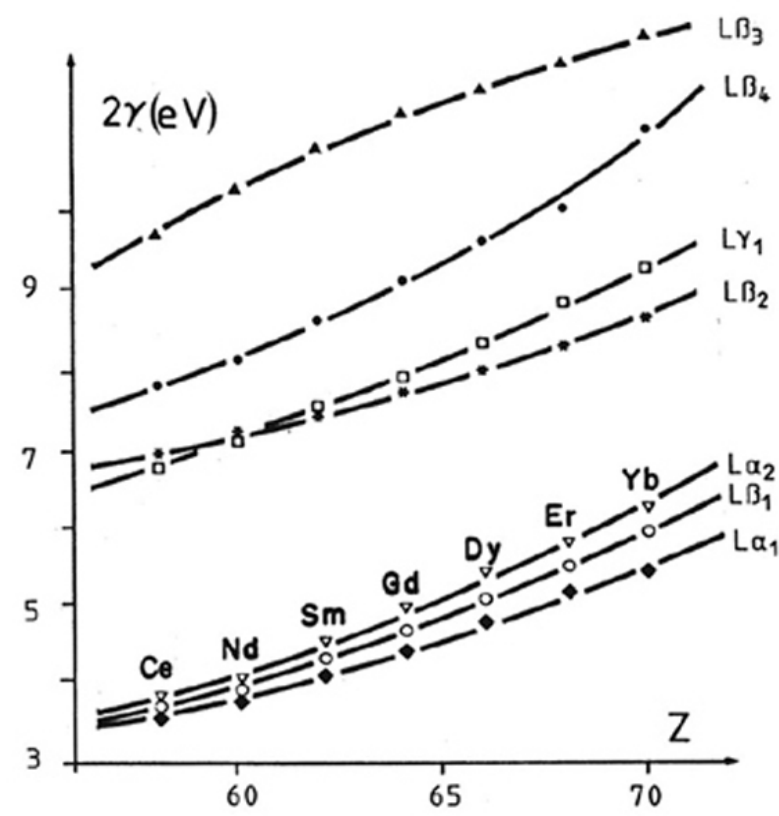

Fig. 17. Natural widths of some L x-ray lines of the rare-earth elements according to Salem and Lee [31].

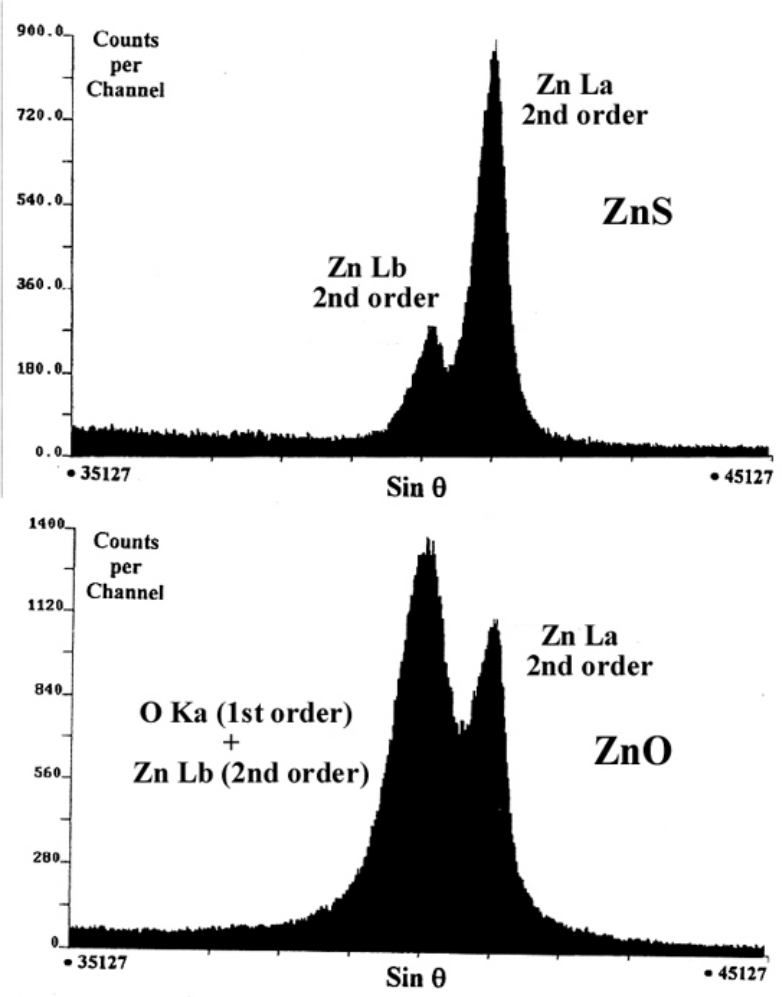

Fig. 18. Interferences between the $\mathrm{O} \mathrm{Ka}$ (first order reflection) and $\mathrm{Zn} \mathrm{L} \alpha, \beta$ emission bands (second order reflection) measured from a $\mathrm{ZnO}$ specimen using a W/Si monochromator. 
To demonstrate this procedure, the fundamental $\mathrm{ZnL} \alpha, \beta$ emission bands for the $\mathrm{ZnO}$ matrix were analyzed with a TAP monochromator by varying the incident energy from $10 \mathrm{keV}$ to $25 \mathrm{keV}$. The energy separation distances between the $\mathrm{ZnL} \alpha, \beta$ diagram bands and their high energy satellites remained constant. The relative intensity ratios $\alpha s / \alpha, \beta s / \beta$, and $\alpha / \beta$ as function of the incident energy are shown in Fig. 19. The theoretical separation distance $[\mathrm{ZnL} \beta-\mathrm{ZnL} \alpha]$ for the diagram lines, and the intensity ratios $\alpha s / \alpha, \beta s / \beta$, and $\beta / \alpha$ derived from Fig. 19 were used as coupled variables in the fitting function describing the interfering $\mathrm{OK} \alpha-\mathrm{ZnL} \alpha, \beta$ emission bands analyzed with the W/Si LMS.

Results of the curve fitting are shown in Fig. 20. As expected because of the peak interferences, the $\mathrm{OK} \alpha$ $k$-ratios for the $\mathrm{ZnO}$ specimen analyzed with the $\mathrm{Fe}_{2} \mathrm{O}_{3}$ standard were in a better agreement with the calculated data when the peak decomposition procedure was used instead of the peak height measurement as illustrated in Fig. 21. A small deviation between the measured and calculated $k$-ratios was still observed. This deviation may result from either uncertainties in some parameters ( mass absorption coefficient, ionization cross-sections, etc.) or from differences in the intrinsic properties of the analyzed specimens.

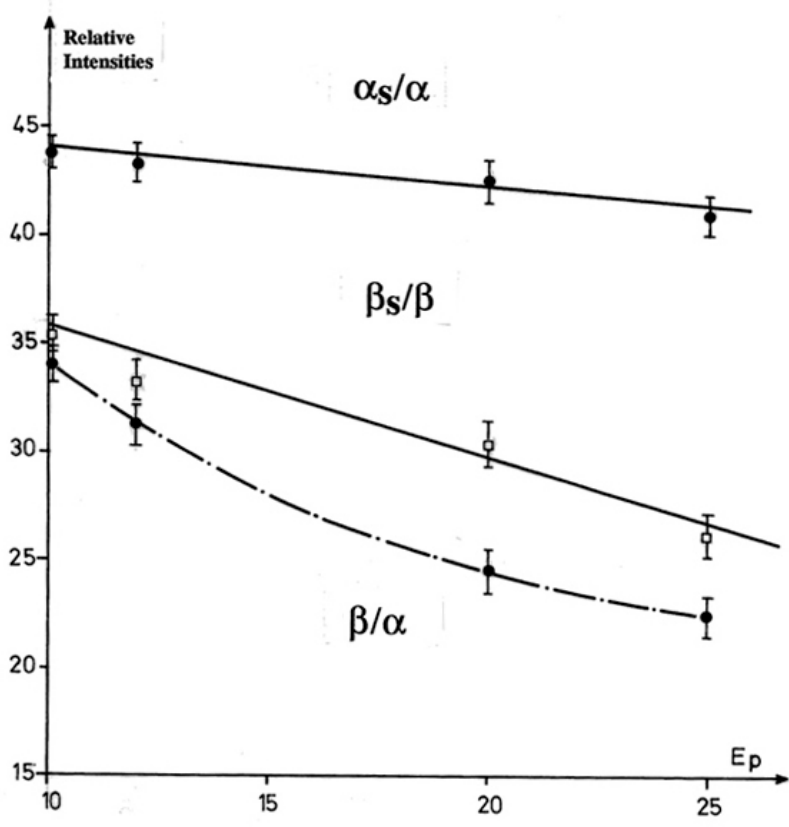

Fig. 19. Parameters describing the $\mathrm{ZnL} \alpha, \beta$ emission bands from a $\mathrm{ZnO}$ specimen measured with a TAP monochromator

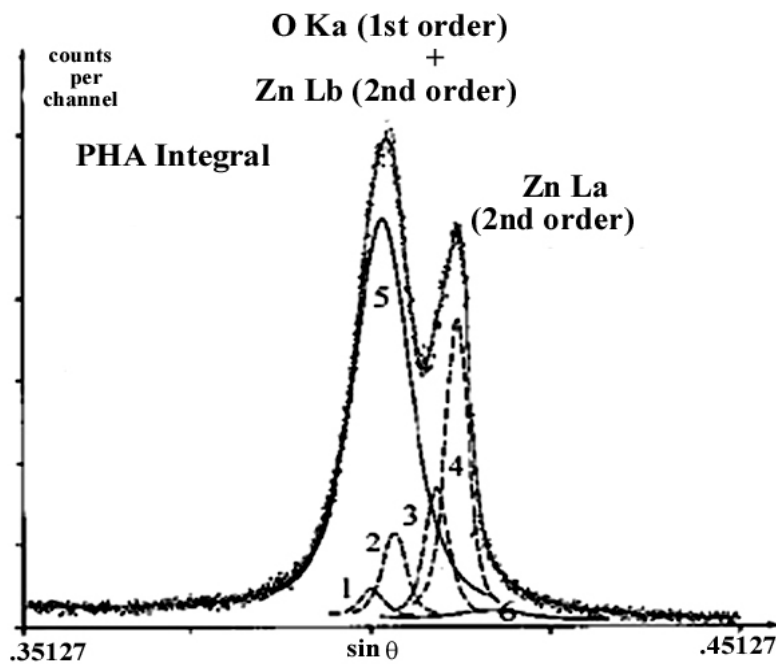

Fig. 20. Results of fit to the $\mathrm{ZnL} \alpha, \beta$ and $\mathrm{OK} \alpha$ emission bands for a $\mathrm{ZnO}$ specimen analyzed with a W/Si multilayer structure as monochromator. The $\mathrm{ZnL} \alpha, \beta$ diagram lines were described by pseudo-Voigt profiles (2)(4) with high energy pseudo-Voigt profiles (1)(3) associated with satellite bands. The parameters describing the $\alpha, \beta, \alpha_{\mathrm{s}}$ and $\beta_{\mathrm{s}}$ components were set as coupled variables according to the data shown in Fig. 19. All parameters in the pseudo-Voigt profiles (5)(6) describing the OK $\alpha$ peak shape were kept as independent variables. The digital spectra were acquired using the integral operating mode of the PHA.

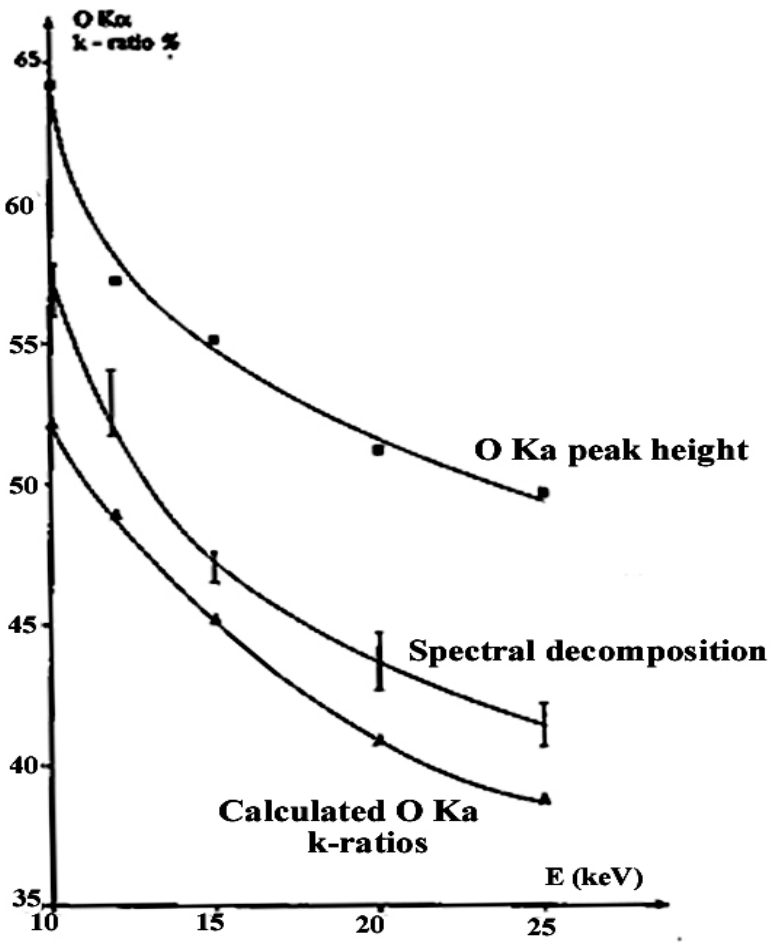

Fig. 21. Variations as a function of the incident energy of the $\mathrm{OK} \alpha$ intensity ratios for a $\mathrm{ZnO}$ specimen with respect to an $\mathrm{Fe}_{2} \mathrm{O}_{3}$ reference specimen. All spectra were acquired using the integral operating mode of the PHA. 


\subsection{Peak Shape Modifications Related to the Presence of Absorption Edges}

\subsubsection{FeL $\alpha, \beta$ Emission Bands From Pure Iron}

The shape of the FeL $\alpha, \beta$ emission spectra also depends on the electron incident energy as illustrated in Fig. 22. The spectra were measured with a TAP monochromator, the incident energy was $3 \mathrm{keV}$ and $7 \mathrm{keV}$. The width at half-maximum (FWHM) increases as the incident energy is decreased from $7 \mathrm{keV}$ to $3 \mathrm{keV}$. However, the $\mathrm{FeL} \alpha$ to the $\mathrm{FeL} \beta$ intensity ratio is higher for a $3 \mathrm{keV}$ incident energy compared with the ratio at $7 \mathrm{keV}$ energy.

The emission spectrum measured with a $3 \mathrm{keV}$ energy was processed using Eq. [17] as a fitting function and assuming a linear variation of the intensity of the continuous emission within the analyzed wavelength domain (Fig. 22a). The low energy side (long wavelength side) of the FeL $\alpha$ peak was correctly described by a pseudo-Voigt profile with $C_{\mathrm{g}}=0.1$ expressing a near Lorentzian shape of the peak. In order to obtain a satisfactory quality of fit to the $L \alpha$ peak a second pseudo-Voigt component of weak amplitude was added on the high energy side (short wavelength side) of the peak. Similarly, two pseudo-Voigt profiles with $C_{\mathrm{g}}=0.1$ were used to described the FeL $\beta$ peak. These high energy components probably correspond to satellite lines resulting from shake-off or Coster-Kronig mechanisms. The results of fit led to a $4.0 \mathrm{eV}$ FWHM for the FeL $\alpha$ peak consistent with the $3.7 \mathrm{eV}$ FWHM value reported by Bonnelle [32]. The near Lorentzian peak shape of the spectrum measured with a $3 \mathrm{keV}$ incident energy indicates that the calculated FWHM values are probably close to those of the natural Lorentzian width and that for the analyzed wavelength region, the TAP monochromator has a small contribution to the observed peak shape. This result is also supported by data previously reported by Rémond et al. [5] studying the $\mathrm{FeL} \alpha, \beta$ emission bands measured with a $15 \mathrm{keV}$ incident energy. For that incident energy, the $F e K \alpha_{1,2}$ ninth order reflection are detected between the FeL $\beta$ and the FeL $\alpha$ peaks. The FWHM of the FeK $\alpha_{1,2}$ peaks were found to be $3.4 \mathrm{eV}$ and $3.8 \mathrm{eV}$, respectively. These values are similar to intrinsic widths reported by Salem and Lee [31], supporting the weak instrumental broadening of the monochromator in the analyzed region.

The FeL $\alpha, \beta$ emission bands measured with a $7 \mathrm{keV}$ incident energy have a narrower FWHM values than for the spectrum measured with the $3 \mathrm{keV}$ energy as shown in Fig. 22b for the FeL $\alpha$ peak of the pure Fe specimen. Consequently, the $\mathrm{FeL} \alpha$ from pure $\mathrm{Fe}$ is no longer described by a single pseudo-Voigt profile. Two pseudo-Voigt profiles must be added to the low energy
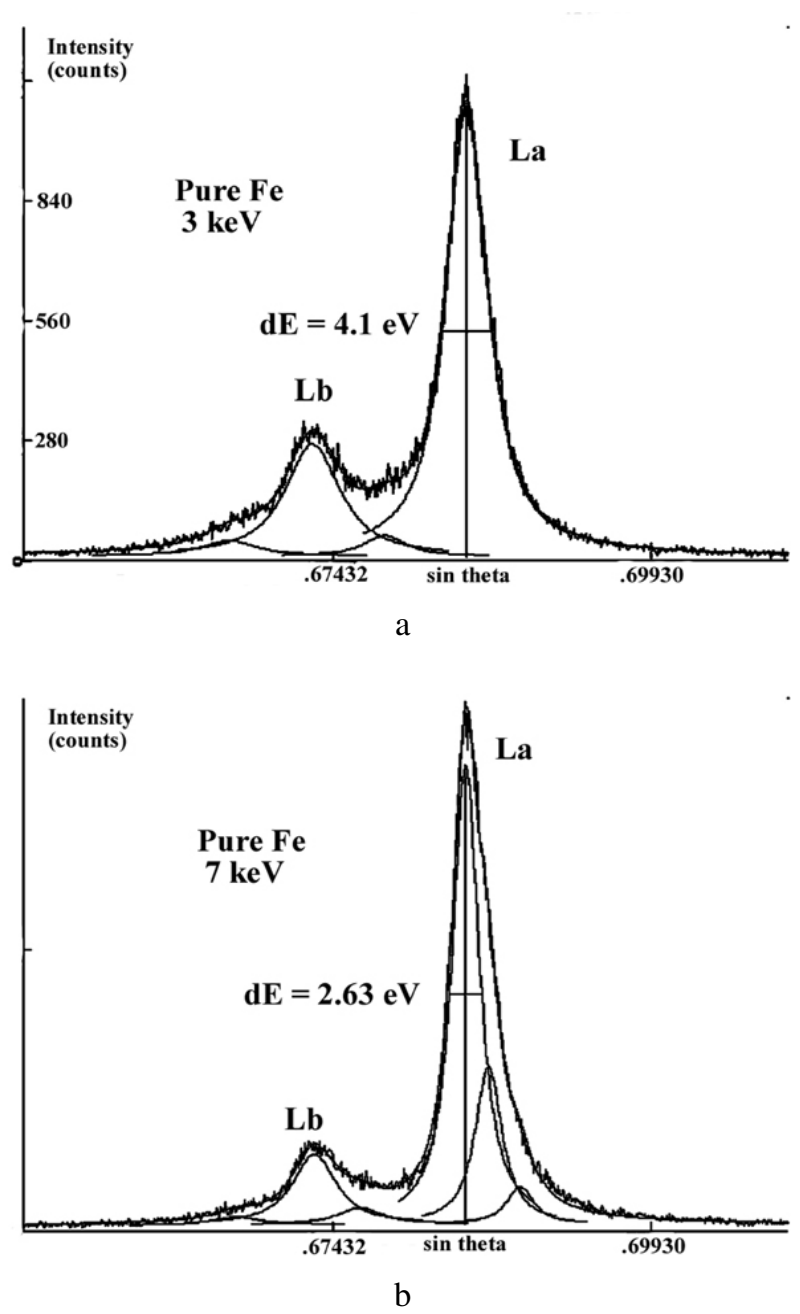

Fig. 22. FeL $\alpha, \beta$ spectra for pure Fe measured with a TAP monochromator and (a ) a $3 \mathrm{keV}$ incident energy and (b) a $7 \mathrm{keV}$ incident energy successively. Spectral decomposition into pseudoVoigt profiles are also show on these figures.

side of the major component but these two offsets have no physical meaning since for a pure metal no low energy satellites are expected with significant amplitudes. The departure from symmetry of the FeL $\alpha$ emission band results from a differential absorption for the high and low energy sides of the peak because the $\mathrm{L}_{3}$ absorption edge intercepts the high energy side of the peak. A similar situation is encountered for the case of the $\mathrm{L}_{2}$ absorption edge which intercepts the high energy side of the L $\beta$ peak.

The experimental spectra measured with a $7 \mathrm{keV}$ incident energy were corrected for self-absorption using the approach described previously which consists in dividing the normalized spectra measured at $3 \mathrm{keV}$ and $7 \mathrm{keV}$ successively resulting in the $g(\chi)$ curve as shown in Fig. 23. Each channel of the experimental spectrum measured with the $7 \mathrm{keV}$ incident energy is divided by 


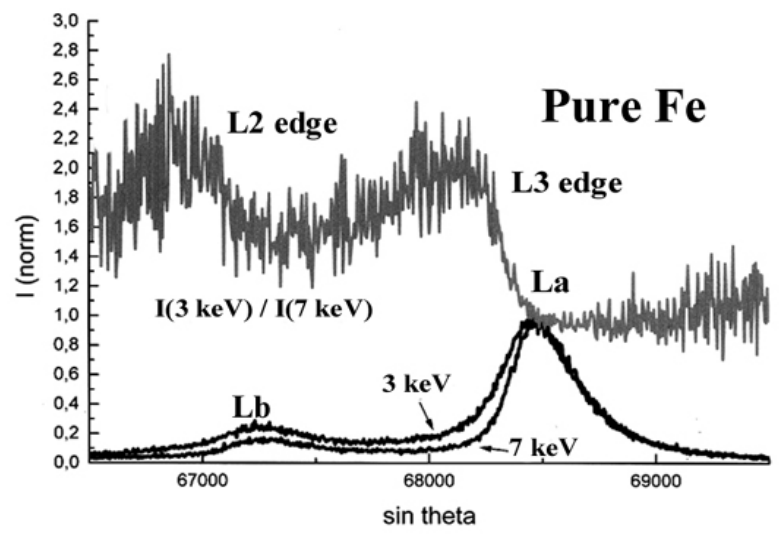

Fig. 23. Absorption correction factor within the wavelength region containing the $\mathrm{FeL} \alpha, \beta$ emission bands from pure Fe.

the value of the $g(\chi)$ curve giving an experimental spectrum whose the shape is corrected for the presence of the absorption edge in the analyzed region. The corrected spectrum is then multiplied channel by channel by the scaling factor in Eq. [21] where $f\left(\chi_{0}\right)$ is calculated using the tabulated mass absorption coefficient of the analyzed elements for the monochromatic $\mathrm{FeL} \alpha$ line. The reconstructed $\mathrm{Fe} L \alpha, \beta$ spectra at $7 \mathrm{keV}$ incident energy is shown in Fig. 24 for the pure iron specimen and was decomposed into pseudo-Voigt profiles, as shown in Fig. 25.

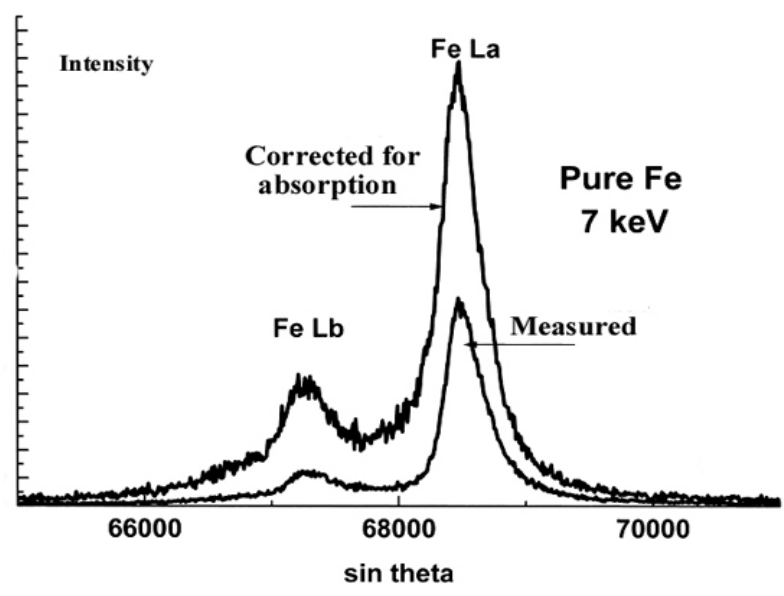

Fig. 24. Reconstructed generated $\mathrm{FeL} \alpha, \beta$ emission spectra in pure $\mathrm{Fe}$ at $7 \mathrm{keV}$ incident energy.

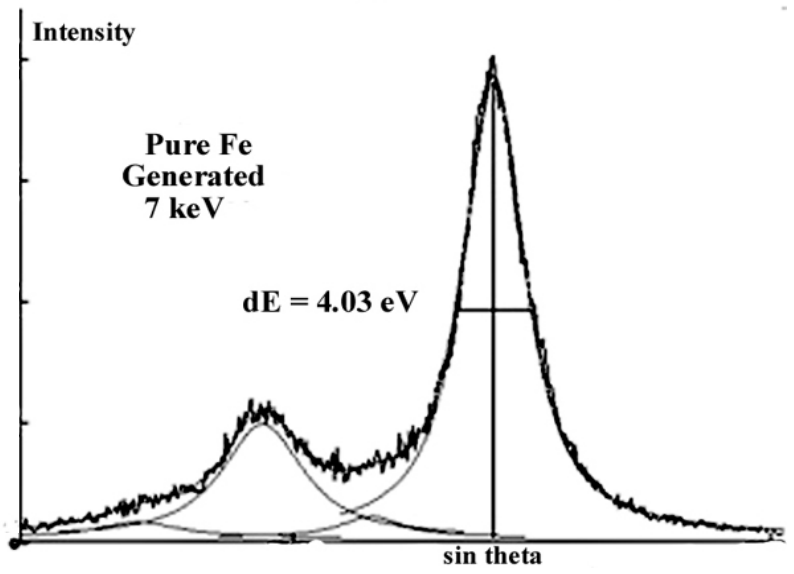

Fig. 25. Spectral decomposition of the generated FeL $\alpha, \beta$ emission spectra at $7 \mathrm{keV}$ incident energy for pure $\mathrm{Fe}$.

\subsubsection{FeL $\alpha, \beta$ Emission Bands From Iron Oxides}

Synthetic $\mathrm{Fe}_{0.94} \mathrm{O}$ (referred below as $\mathrm{FeO}$ ) and natural $\mathrm{Fe}_{2} \mathrm{O}_{3}$ specimens were analyzed according to the experimental conditions mentioned above. As for pure iron, the shape and the peak maximum position depend on the incident energy. The shift of the FeL $\alpha, \beta$ peak position is larger for the iron oxides than for the pure iron specimen. As an example, the shift for pure Fe was $0.18 \mathrm{eV}$ when the incident energy was increased from $3 \mathrm{keV}$ to $7 \mathrm{keV}$ but was $0.8 \mathrm{eV}$ for the $\mathrm{FeO}$ specimen.

The correction curve $g(\chi)$ derived from the normalized spectra measured at $3 \mathrm{keV}$ and $7 \mathrm{keV}$ successively is shown in Fig. 26. In order to obtain the absorption

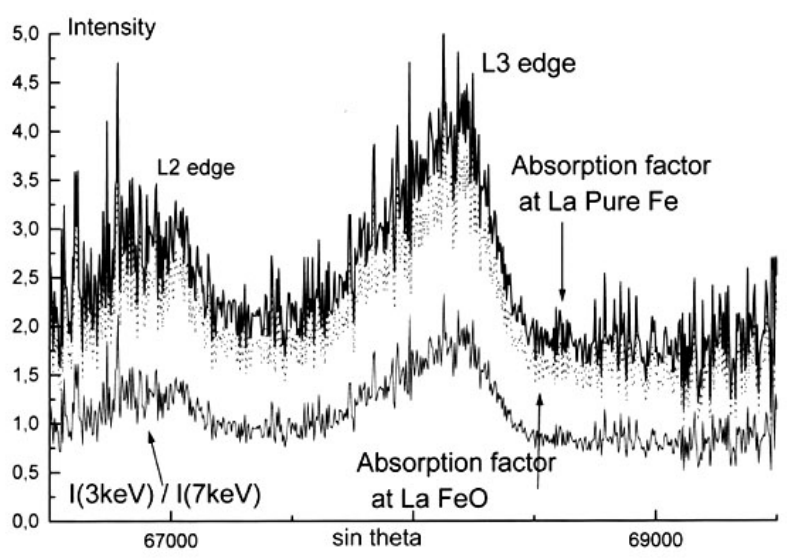

Fig. 26. Absorption correction factors for $\mathrm{FeL} \alpha$ in $\mathrm{FeO}$ within the wavelength region containing the $\mathrm{FeL}$ emission bands. 
correction factor for each analyzed channel, the $g(\chi)$ curve must be multiplied by the scaling factor (Eq. [21]) for the excitation conditions used.

In order to calculate the $f(\chi)$ factor, the mass absorption coefficient associated to the maximum position of the emission bands for the analyzed specimens must be known. In practice, only the mass absorption coefficient for FeL $\alpha$ at the diagram FeL $\alpha$ emission position for pure $\mathrm{Fe}$ is obtained from data Tables. For the iron oxides, the absorption coefficient is calculated by weighting the mass absorption coefficients for FeL $\alpha$ by the mass concentrations of iron and oxygen in the analyzed iron oxide specimens. Figure 26 illustrates the variation of the correction factors within the analyzed wavelength domain when the absorption factor for the monochromatic FeL $\alpha$ line is applied to the maximum position of the pure $\mathrm{Fe}$ or the iron oxide specimens analyzed with a $7 \mathrm{keV}$ incident energy. The corresponding corrected spectra representing the generated intensity of the FeL $\alpha, \beta$ emission bands are shown in Fig. 27 for the $\mathrm{FeO}$ specimen. Similar results are obtained for the $\mathrm{Fe}_{2} \mathrm{O}_{3}$ specimen.

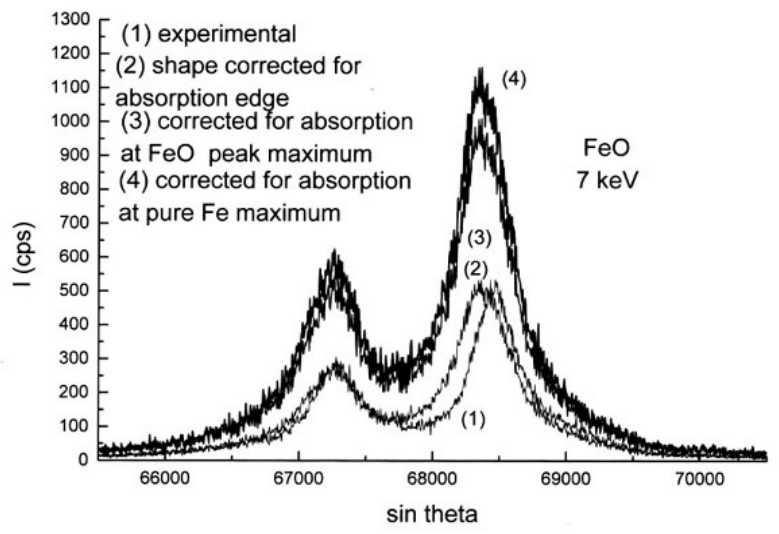

Fig. 27. FeL $\alpha, \beta$ emission spectrum corrected for absorption from an $\mathrm{FeO}$ specimen measured with a $7 \mathrm{keV}$ incident energy.

Spectra corrected for self-absorption were decomposed into the sum of pseudo-Voigt profiles as shown in Fig. 28. Two pseudo-Voigt profiles of equal width were used to describe the L $\alpha$ emission band. The separation distance between the major diagram peak and the low energy band as well as the relative intensity of the two features are different for the $\mathrm{FeO}$ and the $\mathrm{Fe}_{2} \mathrm{O}_{3}$ specimens as shown in Table 1 . The diagram peaks are accompanied by a low energy band probably resulting from bonding states and possibly with a contribution of radiative Auger emission, which can be measured owing to the energy resolution and sensitivity of a WDS as illustrated by Takahashi et al. [34].
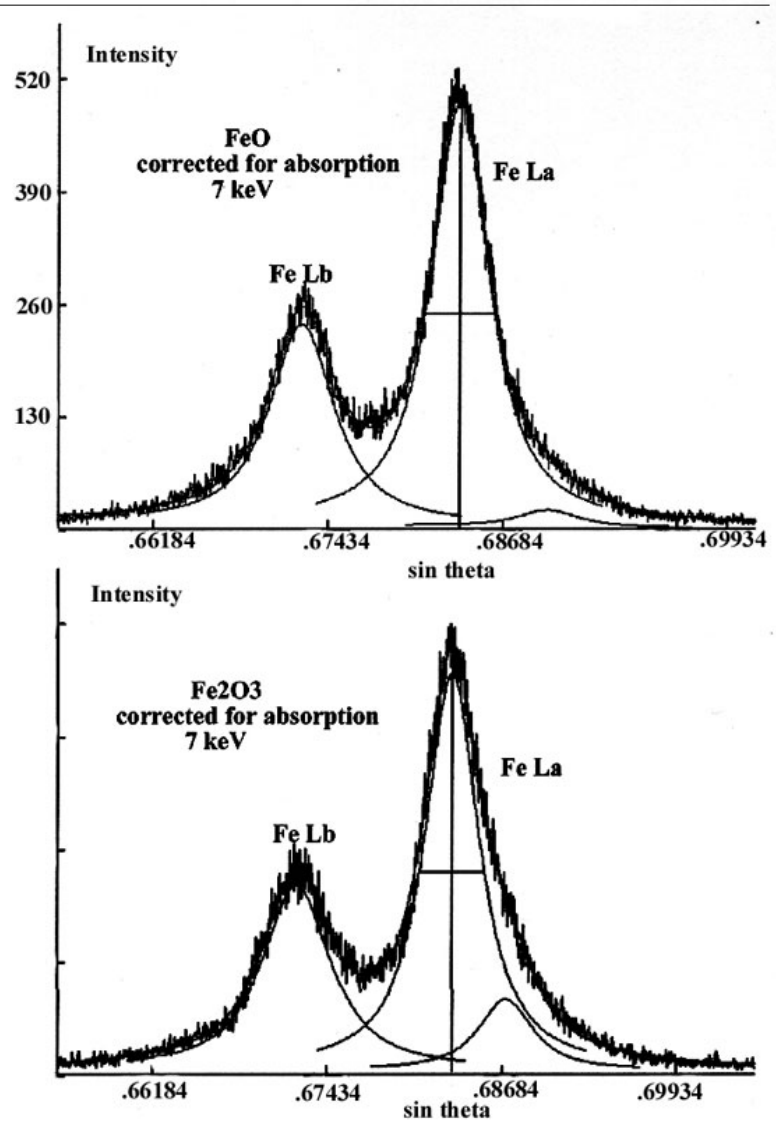

Fig. 28. Decomposition into pseudo-Voigt profiles of spectra corrected for self-absorption in $\mathrm{FeO}$ and $\mathrm{Fe}_{2} \mathrm{O}_{3}$ specimens analyzed with a $7 \mathrm{keV}$ incident energy.

The FeL $\alpha$ intensity from the $\mathrm{FeO}$ specimen was expressed in terms of mass concentration using the $\mathrm{Fe}_{2} \mathrm{O}_{3}$ emission spectrum as a reference. The total area of the experimental FeL $\alpha$ band, i.e., the diagram band and the low energy band, was used for quantitative analysis and the experimental concentration, $k$, was corrected for absorption according to the usual procedure used in EPMA analysis. In a second experiment, the emission spectra measured with a $7 \mathrm{keV}$ incident energy were first corrected for self-absorption before to determine the total peak areas. Thus, the intensities derived from the fits represent the generated intensities. The ratio of the FeL $\alpha$ intensity measured from the corrected spectra for $\mathrm{FeO}$ and $\mathrm{Fe}_{2} \mathrm{O}_{3}$, respectively, directly gives the concentration of iron. Quantitative results are shown in Table 2. Correcting the spectra for non-uniform $f(\chi)$ absorption correction factor within the FeL $\alpha$ emission bands leads to an improvement of the quantitative results compared with those derived from the conventional approach where only the absorption factor associated with the maximum emission of the analyzed $\mathrm{x}$-ray emission is applied. 
Table 1. Spectral decomposition of the FeL $\alpha$ emission corrected for self-absorption (7 keV incident energy)

\begin{tabular}{lcccc}
\hline \hline Specimen & \multicolumn{2}{c}{ L $\alpha$ Diagram band } & \multicolumn{2}{c}{ Low energy band } \\
& Position & $\begin{array}{c}\text { Width } \\
(\mathrm{eV})\end{array}$ & $\begin{array}{c}\text { Position } \\
(\mathrm{eV})\end{array}$ & $\begin{array}{c}\text { Relative } \\
\text { intensity }(\%)\end{array}$ \\
\hline $\mathrm{FeO}$ & 704.3 & 5.4 & 697.7 & 4.0 \\
$\mathrm{Fe}_{2} \mathrm{O}_{3}$ & 704.7 & 4.8 & 700.7 & 15.1 \\
\hline
\end{tabular}

Table 2. Quantitative analysis of $\mathrm{FeO}$ using $\mathrm{Fe}_{2} \mathrm{O}_{3}$ as standard

\begin{tabular}{cccc}
\hline \hline Ep $(\mathrm{keV})$ & Spectra & $C$ & $\Delta C / C \%$ \\
\hline 3 & Measured & 0.760 & -1.3 \\
7 & Measured & 0.592 & -23 \\
7 & Corrected & 0.757 & -1.7 \\
& for edges & & \\
\hline
\end{tabular}

The use of the total area of the emission band as intensity of the analyzed soft x-ray emission still remains questionable. The low energy band associated with the diagram emission band involves transitions from valence electrons, some of them originating from oxygen states. From this study it is not possible to conclude whether the total peak area or that of the different spectral components of a complex x-ray emission spectrum must be used as intensity measurement in the quantitative analysis. In the present study, the low energy band to the diagram emission band was simply described by a pseudo-Voigt profile. This band is probably complex and the pseudo-Voigt profile only represents the envelope of many features. Improvement in the fitting procedure will be performed when theoretical models of the radiative mechanisms will be undertaken. Further study is required on compounds where the same element is present in different valence states in order to account for the differential absorption effect.

The uncertainty in the quantitative data also results from the choice of the mass absorption coefficients to be used for the calculation of the $f(\chi)$ absorption correction factor. However, the use of mass absorption coefficients given for pure elements remains questionable since, owing to the difference in the electronic structure, the mass absorption coefficients for $\mathrm{FeL} \alpha$ for pure iron and the iron oxides are expected to be different.

\section{Conclusion}

The energy resolution of monochromators used with the EPMA usually have a sufficient resolution to observe non-diagram bands occurring either on the short or long wavelength side of the main diagram peak. The observed line shape is the convolution of the natural physical width with the instrumental response function. For x-ray emission lines occurring at low Bragg angles, broadening and asymmetry of the measured $\mathrm{x}$-ray peaks are observed. The shape of an atomic x-ray peak (resulting from transitions only involving core level electrons) or of an $\mathrm{x}$-ray emission band (involving valence electrons) has been described by pseudo-Voigt profiles in a least-squares fitting analysis of experimental WDS spectra which includes analytical description of the observed emission.

Owing to the large number of variables involved in the analytical description of complex observed $x$-ray emission bands, physical constraints must be applied, as illustrated with the line widths, the relative intensities of the spectroscopic components, and the distortion of the peak profiles, resulting from either instrumental factors or from the presence of absorption edges in the analyzed wavelength domain.

In order to add physical constraints in the fitting procedure, a database is particularly important to develop for complex soft x-ray emission spectra. However, it is apparent from this review on WDS peak shape analysis that for soft $\mathrm{x}$-ray, the correction for selfabsorption in the entire wavelength region containing the analyzed emission bands represent an improvement in quantitative analysis using soft $\mathrm{x}$-ray emission peaks.

\section{References}

[1] G. Remond, Ph. Coutures, C. Gilles, and D. Massiot, Analytical description of $\mathrm{x}$-ray peaks. Application to $\mathrm{L} \mathrm{x}$-ray spectra processing of lanthanide elements by means of the electron probe micro-analyzer, Scanning Microsc. 3, 4, 1059-1086 (1989).

[2] M. Fialin and G. Rémond, Electron probe microanalysis of oxygen in strongly insulating oxides, Microbeam Anal. 2, 179-189 (1993).

[3] M. Fialin, G. Rémond, and C. Bonnelle, New developments in electron probe microanalysis of oxygen in wide bandgap oxides. Microbeam Anal. 3, 211-224 (1994).

[4] G. Rémond,C. Gilles, M. Fialin, O. Rouer, R. Marinenko, R. Myklebust, and D. Newbury, Intensity measurement of wavelength dispersive $\mathrm{x}$-ray emission bands: Applications to the soft x-ray region, Mikrochim. Acta, Suppl. 13, 61-86 (1996).

[5] G. F. Bastin and H. J. M. Heijligers, Quantitative electronprobe microanalysis of ultra-light elements, J. Microsc. Spectrosc. Electron. 11, 215-228 (1986).

[6] G. F. Bastin and H. J. M. Heijligers, Quantitative electron probe microanalysis of ultra-light elements (Boron-Oxygen). Electron Probe Quantitation, K. F. J. Heinrich and D. E. Newbury, eds., Plenum Press, New-York and London, pp. 145-162.

[7] M. Fialin, J. Henoc, F. Maurice, and G. Rémond. Quantitative analysis using soft $\mathrm{x}$-ray spectrometry with the electron probe microanalyzer. 11th Pfefferkorn Conference, Scanning Microscopy, Suppl. 7 (1993).

[8] P. Jonnard, C. Bonnelle, G. Blaise, G. Rémond, and C. Roques-Carmes, $\mathrm{F}+$ and $\mathrm{F}$ centers in $\alpha-\mathrm{Al}_{2} \mathrm{O}_{3}$ by electron induced $\mathrm{x}$-ray emission spectroscopy and cathodoluminescence. J. Appl. Phys. 88 (11), 6413-6417 (2000). 
[9] L. G. Parratt, K $\alpha$ satellites, The Phys. Rev. 50, 1-15 (1936).

[10] L. G. Parratt, Electronic band structure of solids by x-ray spectroscopy, Rev. Mod. Phys. 31 (3) 616-645 (1959).

[11] C. A. Randall and L. G. Parratt. L? satellite lines for elements Mo(42) to Ba(56). Phys. Rev. A 57, 786-791 (1940).

[12] T. Aberg, Theory of multiple ionization processes, Proc. Int. Conf. Inner Shell Ionization Phenomena, USAEC Conf. 720404 USA EC, Atlanta, GA, (1927) pp. 1509-1542.

[13] D. Fabian, Soft X-ray band emission from solids. CRC Critical Reviews in Solid State Sciences, CRC Press, 255-316 (1971).

[14] O. Keski-Rakhonen and J. Ahopelto, The K-M ${ }^{2}$ radiative Auger effect in transition metals. J. Phys. C: Solid State Phys. 13, 471-482 (1980).

[15] S. I. Salem and B. L. Scott, Splitting of the $4 d 3 / 2$ and $4 d 5 / 2$ levels in rare-earth elements and their oxides. Phys. Rev. A 13, 330-334 (1974).

[16] K. Tsutsumi, H. Nakamori, and K. Ichikawa, X-ray MnK $\beta$ emission spectra of manganese oxides and manganates. Phys. Rev. B 13, 929-933 (1976).

[17] K. S. Srivastava, A. K. Srivastava, K. S. M. Husain, and S. Singh, Electron-electron interaction in the X-ray emission spectra of rare-earth elements and their oxides, Indian J. Pure Appl. Phys., 21, 256-257 (1983).

[18] G. Rémond, J. L. Campbell, R. H. Packwood., and M. Fialin, Spectral decomposition of wavelength dispersive $\mathrm{x}$-ray spectra: Implications for quantitative analysis in the electron probe microanalyzer, Scanning Microsc., Suppl. 7, 89-132 (1993).

[19] Y. Cauchois and C. Bonnelle, X-ray diffraction spectrometry. Atomic Inner-Shell Processes, Tome II: Experimental Approaches and Applications, B. Crasemann, ed., Academic Press (1975) pp. 84-121.

[20] C. G. Darwin, Y. Cauchois, and C. Bonnelle, X-ray diffraction spectrometry. Atomic Inner-Shell Processes, Tome II: Experimental Approaches and Applications, B. Crasemann, ed., Academic Press (1975) 84-121.

[21] R. A. Mattson and R. C. Ehlert, The application of a soft x-ray spectrometer to study the oxygen and fluorine emission lines from oxides and fluorites. Adv. X-Ray Anal. 9, 471-486 (1966).

[22] P. G. Self, K. Norrish, A. R. Milnes, J. Graham, and B. Robinson, Holes in the background in XRS, X-Ray Spectrom. 19, 59-61 (1990).

[23] G. Rémond, R. H. Packwood, and C. Gilles, Trace element standards for the electron microanalyser using layered and ion implanted materials. Analyst 120, 1247-1260 (1995).

[24] D. A. Wollman, K. D. Irwin, G. C. Hilton, L. L. Dulcie, D. E. Newbury, and J. M. Martinis, High-resolution, energy-dispersive microcalorimeter spectrometer for $\mathrm{x}$-ray microanalysis, $\mathrm{J}$. Micros. 188, 196-223 (1997).

[25] G. W. Phillips and K. W. Marlow, Automatic analysis of gammaray spectra from germanium detectors. Nucl. Instr. Meth. 137, 525-536 (1976).

[26] J. L. Campbell, A. Perujo, and B. M. Millman, Analytic description of $\mathrm{Si}(\mathrm{Li})$ spectral line shapes due to monoenergetic photons, X-Ray Spectrom. 16, 195-201 (1987).

[27] T. C. Huang and G. Lim, Resolution of overlapping X-ray fluorescence peaks with the pseudo-Voigt function. Adv. X-Ray Anal. 29, 461-468 (1986).

[28] D. G. W. Smith and S. J. B. Reed, The calculation of background dispersive electron microprobe analysis, X-Ray Spectrom. 10, 4, 159-162 (1981).

[29] D. Massiot, Shape comparison of physical spectra: Application to Mössbauer spectra of silicate glasses. J. Non-Crystalline Solids 69, 371-380 (1985).
[30] A. Laakkonen and G. Graeffe, M x-ray linewidths of gold. J. Phys. (Paris), Colloque C9, 212, 48, 605-608 (1987)..

[31] S. I. Salem and P. L. Lee, Experimental widths of x-ray lines, At. Data Nucl. Data Tables 18, 234-241 (1976).

[32] C. Bonnelle, Contribution à l'étude des métaux de transition du premier groupe, du cuivre et de leurs oxydes par spectrométrie $\mathrm{X}$ dans le domaine de 13 à $22 \AA$ (Contribution to the study of the first series metals, copper and oxides by means of X-ray spectrometry in the 13 to $22 \AA$ ). . Thesis University of Paris, Masson and Co. (eds), Paris, France (1966).

[33] M. Fialin, C. Wagner, and G. Rémond, X-ray emission valence band spectrometry: Application to $\mathrm{Cu}$ and $\mathrm{Fe} \mathrm{L}$ series. EMAS'98, Electron Probe Microanalysis Today, X. Llovet, C. Merlet, and F. Salvat, eds., University of Barcelona (1998) pp. 129-140.

[34] H. Takahashi, I. Harrowfield, C. MacRae, N. Wilson, and K. Tsutsumi, Extended x-ray emission fine structure and high energy satellite lines state measured by electron probe microanalysis, Surface Interface Anal. 31, 118-125 (2001).

About the authors: Guy Rémond is Docteur ès Science (Physique), now retired. Formerly with bureau de Recherches Géologiques et Minières in Orléans (France) as Research Engineer involved in the development of analytical methods and techniques for the microcharacterization of material minerals. In 1995 he joined the Laboratoire de Microanalyse des Surfaces at the Ecole Nationale de Mécanique et des Microtechniques (Besançon, France) and has been involved in a joint research program with the Australian Key centre for Microscopy and Microanalysis (Sydney, Australia). His current research activity includes soft $x$-ray spectrometry at nanoscale and low accelerating voltages for the study of insulating materials.

Robert Myklebust retired from the Microanalysis Group of NIST in 1997 after a 34 year career with the Federal Government, 30 years of which were spent at NBS/NIST. His research interests were centered on $x$-ray spectrometry and related analysis methods: $x$-ray fluorescence, electron probe $x$-ray microanalysis, and energy dispersive $x$-ray spectrometry. Throughout his career, he concentrated on the incorporation of laboratory-scale computation facilities to provide real time computer-aided analysis. He was the principal architect of many of the computer software analytical systems developed in the Microanalysis Research Group: Multi-8, FRAME, COR, FRAMEC, and the Microanalysis Monte Carlo Electron Trajectory Simulator.

Michael Fialin is a PhD in Solid State Physics and is a member of the Board of the French Association for Scanning Electron Microscopy and Analyses. He is a Research Engineer at the Centre National de la Recherche Scientifique (CNRS) in Paris (France). His activity is in the field of $x$-ray spectrometry applied to the micro-characterization of insulating materials 
emphasizing the interpretation and processing of soft $x$-ray spectra.

Clive Nockolds a PhD, now retired, was formerly with the Electron Microscopy Unit at the University of Sydney (Australia). He has over 30 year experience in $x$-ray microanalysis of materials and is interested in problems related to the $x$-ray microanalysis of insulators in the variable pressure SEM.

Matthew Phillips is a Professor and Director of the Microstructural Analysis Unit located at the University of Technology of Sydney (Australia). He has extensive experience in a broad range of microanalysis techniques used to characterize the physical properties of wide band gap semiconductors and insulators. His current research work involves projects investigating the influence of point defects and light element impurities $(H, C$, and $O)$ on the optical and electrical properties of new optoelectronic materials.

Claude Roques-Carmes is a Professor at the Ecole Nationale Supérieure de Mécanique et des Microtechniques in Besançon (France) where he created, in 1980 the Laboratoire de Microanalyse des surfaces. The research and development activity of the laboratory is focused on the microcharacterization of surfaces with a particular attention to the description of the topography, to the tribology and the mechanics of surface structures and to the reactivity of surfaces successively. Models are developed for the understanding of the transfer of energy and material at the interfaces. 